\title{
HELECHOS Y LICOFITOS EPÍFITOS DE VERACRUZ, MÉXICO: RIQUEZA Y DISTRIBUCIÓN
}

\author{
Aniceto Mendoza-Ruiz ${ }^{1,2}$, Jacqueline Ceja-Romero ${ }^{1}$ y Blanca Pérez-García ${ }^{1}$ \\ ${ }^{1}$ Universidad Autónoma Metropolitana-Unidad Iztapalapa, Área de Botánica \\ Estructural y Sistemática Vegetal, Departamento de Biología, Apdo. postal 55-535, \\ 09340, México, D.F., México. \\ 2Autor para la correspondencia: amr@xanum.uam.mx
}

\section{RESUMEN}

Con base en la revisión de material de herbario y literatura especializada, así como en la recolección de ejemplares botánicos, obtuvimos la información correspondiente a la riqueza, distribución por municipio, tipo de vegetación e intervalo altitudinal de los helechos y licofitos epífitos que habitan en el estado de Veracruz. Se registraron para el área de estudio 11 familias, 33 géneros, 173 especies con al menos un ejemplar de respaldo y 23 citadas en la literatura. Las familias mejor representadas a nivel específico fueron Polypodiaceae, Hymenophyllaceae y Dryopteridaceae, mientras que los géneros con mayor número de taxa fueron Polypodium, Asplenium, Elaphoglossum y Trichomanes. La mayoría de las plantas se distribuyen en los bosques mesófilo de montaña, tropical perennifolio y de Quercus, en un intervalo altitudinal que va de los 751 a los $1750 \mathrm{~m}$.

Palabras clave: bosque mesófilo de montaña, bosque tropical perennifolio, distribución, diversidad, intervalo altitudinal, pteridofitas epífitas.

\section{ABSTRACT}

Based on the review of herbarium material and specialized literature, as well as recollection of botanical specimens, we obtained information about the species richness, municipal distribution, vegetation type and altitudinal range of epiphytic ferns and lycophytes that occur in the state of Veracruz, Mexico. We recorded 11 families, 33 genera and 173 species with at least one herbarium voucher, and 23 cited in the literature. The families with highest species number were Polypodiaceae, Dryopteridaceae and Hymenophyllaceae, 
whereas the genera with most taxa were Polypodium, Asplenium, Trichomanes and Elaphoglossum. Most plants are distributed in cloud forest, tropical evergreen forest and oak forest, in an altitudinal range from 751 to $1750 \mathrm{~m}$.

Key words: altitudinal range, cloud forest, distribution, diversity, epiphytic pteridophytes, tropical evergreen forest.

\section{INTRODUCCIÓN}

Se calcula que entre 25 y $29 \%$ de las especies de helechos y licofitos (pteridofitas sensu lato) en el mundo son epífitas, por lo que, junto con las familias Orchidaceae y Bromeliaceae, constituyen uno de los grupos de plantas vasculares en donde se concentra la mayor riqueza de taxa con esta forma de vida (Kress, 1986, 1989; Benzing, 1990; Zotz, 2013). En algunas selvas tropicales de Australia, Nueva Zelanda e islas de la Micronesia, llegan a representar entre 36 y $72 \%$ de la diversidad epifítica (Dubuisson et al., 2009).

En México se tienen registradas 1030 especies de pteridofitas (Mickel y Smith, 2004; Tejero-Díez et al., 2014), de las cuales 265 presentan algún tipo de epifitismo. Se distribuyen principalmente en los bosques mesófilos de montaña, selvas tropicales y bosques de pino-encino del país (Mickel y Smith, 2004). Veracruz es el estado que, después de Chiapas y Oaxaca, cuenta con el mayor número de plantas vasculares de México, con 7855 especies (Castillo-Campos et al., 2011), de las cuales 565 son pteridobiontes (Tejero-Díez et al., 2011; Krömer et al., 2013a). Además, es una de las entidades que cuenta con mayor diversidad de tipos de vegetación en el país (Castillo-Campos et al., 2011), incluyendo aquellos en los que se desarrollan de manera favorable las epífitas.

Son numerosos los trabajos florísticos y ecológicos que sobre los distintos grupos de plantas con representantes epífitos se han realizado para el estado de Veracruz, algunos de ellos dirigidos particularmente a familias como Orchidaceae y Bromeliaceae (p. ej. García-Cruz y Sánchez-Saldaña, 1999; Alanís-Méndez et al., 2007; García-Franco y Toledo-Aceves, 2008; Cortés-Flores, 2009; Morales-Linares, 2009; Espejo-Serna y López-Ferrari, 2011) y otros encaminados a estudiar los factores que determinan la distribución y abundancia de los taxa, incluidas las pteridofitas, que presentan esta forma de vida (Hietz y Hietz-Seifert, 1995; García-Franco, 1996; Hietz y Briones, 1998, 2004; Flores-Palacios y García-Franco, 2001, 2004, 2006, 2008; Flores-Palacios, 2003; Hietz, 2005; Mehltreter et al., 2005; CarreñoRocabado, 2006; Rojas-Santiago, 2012; Krömer et al., 2013a, b). 
Con referencia a helechos y licofitos, existen trabajos que proporcionan información sobre su diversidad y distribución en algunas regiones y/o tipos de vegetación, pero no consideran la forma de vida (Zamora-Crescencio y Castillo-Campos, 1997; Acosta-Rosado, 2002; Castillo-Campos et al., 2003; Vázquez-Torres et al., 2006; García-Franco et al., 2008; Mehltreter, 2008; Castillo-Campos y Luna-Monterrojo, 2009; Gómez-Ortega, 2010), mientras que otros sí la incluyen. De estos últimos se hace un breve recuento a continuación.

Valdivia (1977) presenta el número, abundancia y características ecológicas de las epífitas vasculares de la región del río Uxpanapa, para la que registra 153 especies, de las cuales 32 son pteridofitas. Para la misma zona, Riba y Pérez-García (1979) presentan una lista de 69 especies de helechos, 28 de ellas epífitas. Lira (1983) y Lira y Riba (1984) en la flora pteridológica de la Sierra de Santa Marta reportan 66 especies con hábito epífito. Bongers et al. (1988) analizan la fisonomía, estructura y composición florística de una hectárea de Los Tuxtlas y cuantifican 58 especies epífitas, seis de ellas helechos. Luna et al. (1988) hacen el estudio florístico de tres localidades con bosque mesófilo de montaña en Teocelo y mencionan como epífitas a Polypodium angustifolium Sw. [=Campyloneurum angustifolium (Sw.) Fée] y Polypodium aureum L. [=Phlebodium pseudoaureum (Cav.) Lellinger]. Palacios-Ríos (1992) en su tesis sobre las pteridofitas del estado de Veracruz enlista 572 taxa, de los cuales 106 son holoepífitos. Mehltreter et al. (2005) comparan la diversidad, abundancia y frecuencia de las epífitas vasculares que crecen sobre las angiospermas y helechos arborescentes de un bosque mesófilo de montaña en San Andrés Tlalnelhuayocan e indican la presencia de 24 especies de helechos con esta forma de vida. Pérez-Peña (2007) estudia la diversidad de epífitas vasculares en 21 parcelas con tres tipos de vegetación, en terrenos cercanos a la Estación Biológica de Los Tuxtlas y enumera un total de 81 especies, de las cuales 21 son pteridofitas. Flores-Méndez (2008) en su trabajo sobre epífitas vasculares del bosque mesófilo de montaña en La Quinta, en el municipio de Zongolica, encuentra 94 especies, 29 de ellas pteridofitas. Flores-Palacios y García-Franco (2008) analizan el efecto del aislamiento del hábitat en la diversidad de epífitas vasculares en tres parcelas de un fragmento de bosque en San Andrés Tlalnelhuayocan, de los 118 taxa presentes, 38 son helechos. Viccon-Esquivel (2009), en su escrito sobre la riqueza y composición florística de las epífitas vasculares del bosque mesófilo de montaña de Atzalan y Zongolica, reporta 87 especies (49 pteridofitas) para el primero y 93 (39 pteridofitas) para el segundo; señala además que los helechos son el principal grupo de epífitas, representando 46\% de la riqueza total. Salazar-Rodríguez (2010) en su trabajo sobre las holoepífitas vasculares del bosque mesófilo de montaña del municipio de Acajete, incluye 114 especies, de las que 41 son pteridofitas. Gómez-Pompa y Castillo- 
Campos (2010), en el Atlas de la flora de Veracruz, describen dentro de lo que llaman las especies notables a Polypodium eatonii y Psilotum complanatum, ambas epífitas. Gómez-Díaz (2010) compara la diversidad de epífitas presentes en un bosque mesófilo de montaña y un acahual en el municipio de Tlalnelhuayocan, obteniendo un inventario de 95 especies, 34 de ellas pteridofitas. Flores-Palacios y Valencia-Díaz (2007) y Flores-Palacios et al. (2011) monitorearon los puntos de venta de plantas en el área de Xalapa-Coatepec en los que contabilizaron 207 especies de epífitas, 63 de las cuales son plantas vasculares sin semilla. Tejero-Díez et al. (2011) registran para Veracruz 557 especies, tres variedades y dos híbridos de helechos y licopodios, hacen el análisis de su distribución por tipo de vegetación, región topográfica, estado de conservación, forma de vida y señalan la presencia de un elevado número de plantas epífitas (27\%). Krömer et al. (2013a) actualizan la información que se tiene sobre taxonomía, distribución y estado de conservación de la familia Grammitidaceae en el estado, reconocen 18 especies, 15 de ellas epífitas. Krömer et al. (2013b), en su análisis sobre el efecto de la altitud y el clima en la riqueza de especies, reportan para Los Tuxtlas 108 especies de helechos, 61 (56.5\%) de ellas con hábito epífito. Carvajal-Hernández et al. (2014) enlistan para la Sierra de Chiconquiaco del centro de Veracruz un total de 81 especies de pteridobiontes, de las cuales 26 (32\%) son epífitas.

Dado que la información que se tiene sobre los helechos y licofitos epífitos de Veracruz se encuentra dispersa, este trabajo tiene como objetivo integrar y complementar el conocimiento que sobre estos dos grupos de plantas se ha generado hasta el momento. Se presenta la riqueza de especies, composición florística, distribución por municipio, tipo de vegetación e intervalo altitudinal.

Área de estudio

El estado de Veracruz tiene una extensión territorial de $71820 \mathrm{~km}^{2}$, lo que representa $3.7 \%$ de la superficie total de México. Se localiza entre los $17^{\circ} 09^{\prime}$ y $22^{\circ} 28^{\prime}$ de latitud norte y los $93^{\circ} 36^{\prime}$ y $98^{\circ} 39^{\prime}$ de longitud oeste. Colinda al norte con el estado de Tamaulipas, al este con el Golfo de México y el estado de Tabasco, al sureste con el estado de Chiapas, al sur con el estado de Oaxaca y al oeste con los estados de Puebla, Hidalgo y San Luis Potosí. Está dividido en 212 municipios (INEGI, 2012a, 2012b).

Fisiografía

En la entidad confluyen siete provincias fisiográficas: 1. Sierra Madre Oriental, subprovincia Carso Huasteco (con 4.89\% de la superficie del estado); 2. Llanura Costera del Golfo Norte, subprovincia Llanuras y Lomeríos (30.05\%); 3. Eje Neovol- 
cánico, subprovincias de Chiconquiaco (7.91\%) y de Lagos y Volcanes de Anáhuac (2.94\%); 4. Sierra Madre del Sur, subprovincia Sierras Orientales (2.78\%); 5. Llanura Costera del Golfo Sur, subprovincias Llanura Costera Veracruzana (43.02\%), Llanura y Pantanos Tabasqueños $(0.76 \%)$ y Sierra de los Tuxtlas $(4.19 \%)$; 6. Sierra de Chiapas y Guatemala, subprovincia Sierras del Norte de Chiapas (2.83\%) y 7. Cordillera Centroamericana, subprovincia Sierra del sur de Chiapas (0.63\%) (INEGI, 2012a, 2012b).

Las principales elevaciones son: Volcán Pico de Orizaba (5610 m), Volcán Cofre de Perote (4200 m), Cerro Tepozteca (3140 m), Cerro Cuamila (2980 m), Volcán San Martín Tuxtla (1680 m), Sierra de Santa Martha (1200 m), Cerro San Martín (1160 m), Sierra Otontepec (1160 m) y Sierra La Garganta (800 m) (Anónimo, 2013).

\section{Hidrología}

Los recursos hídricos de Veracruz están conformados por una red de ríos perennes, intermitentes y arroyos que fluyen a una amplia planicie costera, donde se ubican al menos 16 lagunas costeras, así como cuatro ríos o estuarios que descargan directamente a la zona marina adyacente. Se ha estimado que el escurrimiento anual superficial que ocurre en el estado representa casi 30\% del total nacional (Pérez-Maqueo et al., 2011). De las cinco regiones hidrológicas que se reconocen en la entidad, destacan por la magnitud de su aportación las de Coatzacoalcos y Papaloapan, con 14\% y 13\% del escurrimiento total del estado respectivamente (Pérez-Maqueo et al., 2011).

\section{Clima}

La ubicación latitudinal y la variación altitudinal presentes en el estado de Veracruz hacen que las condiciones de temperatura y humedad sean muy diversos (Soto-Esparza y Giddings-Berger, 2011), lo que permite el desarrollo de distintos tipos y subtipos climáticos. El cálido húmedo (31.5\% de la superficie del estado) y el cálido subhúmedo (54.1\%) se distribuyen principalmente en las llanuras costeras del Golfo, a una altitud máxima de $1000 \mathrm{~m}$, la temperatura media anual varía desde 22 a $26^{\circ} \mathrm{C}$ y la precipitación total anual de 2000 a poco más de $3500 \mathrm{~mm}$ (INEGI, 2012a), aunque para la región de Los Tuxtlas se han registrado hasta $4201 \mathrm{~mm}$ (GutiérrezGarcía \& Ricker, 2011). El semicálido húmedo (8.7\%) se presenta en sitios con una altitud de 1000 a $1600 \mathrm{~m}$, la temperatura media es de 18 a $22{ }^{\circ} \mathrm{C}$ y la precipitación total anual de 2000 a más de $2500 \mathrm{~mm}$. El templado húmedo (3.34\%) y templado subhúmedo (1.38\%) se encuentran en zonas con altitudes entre 1600 y $2800 \mathrm{~m}$, la temperatura media anual oscila de 12 a $18{ }^{\circ} \mathrm{C}$ y la precipitación total anual de 500 a $2500 \mathrm{~mm}$. El semifrío subhúmedo $(0.50 \%)$ se localiza entre los 2800 y $3800 \mathrm{~m}$ 
s.n.m., en el Cofre de Perote y el Pico de Orizaba, respectivamente, la temperatura media va de 5 a $12^{\circ} \mathrm{C}$ y la precipitación total anual de 600 y $1200 \mathrm{~mm}$. El semiseco templado $(0.45 \%)$ se presenta en los alrededores de la ciudad de Perote y al oeste de la Huasteca, su temperatura media anual es de $14{ }^{\circ} \mathrm{C}$ y la precipitación total anual está entre 400 a 500 mm (INEGI, 2012a).

\section{Vegetación}

Las características fisiográficas del estado se reflejan en un amplio gradiente altitudinal, topográfico y climático, lo que ha permitido el desarrollo de 19 tipos de vegetación (Gómez-Pompa y Castillo-Campos, 2010). Sin embargo, la perturbación de los mismos ha ocasionado que probablemente varios de ellos estén por desaparecer (Castillo-Campos et al., 2011). Entre los principales tipos se encuentran: A) bosques de coníferas, con 74003.6 ha (INEGI, 2012a), localizados principalmente en la zona alta y fría del cofre de Perote y del Pico de Orizaba, pero también en otras regiones del estado (Castillo-Campos et al., 2011). B) bosques de encino, ocupan 12 886.5 ha (INEGI, 2012a), se dividen en templados y cálidos, los primeros asociados a pinos (constituyendo bosques de pino-encino) o a bosques mesófilos de montaña, y los segundos a los palmares del centro del estado (Gómez-Pompa y Castillo-Campos, 2010; Castillo-Campos et al., 2011). C) bosques mesófilos de montaña, con 56245.1 ha (INEGI, 2012a), desarrollados principalmente en terrenos accidentados, cañadas, barrancas o laderas con pendiente pronunciada, se caracterizan por su alta humedad durante todo el año, siendo frecuente la presencia de neblina, lo que favorece el crecimiento de epífitas (Gómez-Pompa y Castillo-Campos, 2010; Castillo-Campos et al., 2011). D) selva perennifolia, ocupa 136610.6 ha (INEGI, 2012a), cubría grandes extensiones de áreas planas de baja altitud; sin embargo, la deforestación las ha reducido a solo algunos manchones, de los cuales los que se localizan en las regiones de Los Tuxtlas y Uxpanapa son representativos. E) selva caducifolia, con 5410.7 ha (INEGI, 2012a), presente principalmente en el centro y norte del estado, en las zonas con clima cálido subhúmedo con lluvias de verano y baja precipitación; es tolerante a la perturbación (Gómez-Pompa y Castillo-Campos, 2010; Castillo-Campos et al., 2011). F) matorral xerófilo, con 12722.9 ha (INEGI, 2012a), localizado en el sotavento del Cofre de Perote, en los límites con el estado de Puebla; generalmente se ocupa para el pastoreo de ganado caprino. G) otros tipos de vegetación, entre ellos sobresalen el pastizal cultivado que ha reemplazado a comunidades vegetales originales y que ocupa una extensa superficie, así como el manglar, localizado en forma de manchones a todo lo largo de la costa veracruzana. También están presentes el tular, el palmar, el popal y la vegetación acuática. 


\section{MATERIAL Y MÉTODOS}

Durante los años 2006 a 2014, se llevó a cabo la recopilación y consulta de los trabajos taxonómicos, florísticos y de vegetación relacionados con el tema y se revisaron las siguientes colecciones institucionales mexicanas: CORU, ENCB, FCME, IEB, MEXU, OAX, UAMIZ, XAL y XALU (Thiers, B., continuamente actualizado). Además se consultaron vía internet las bases de datos del University of Arizona herbarium (ASU), Digital specimen images of Herbarium Berolinense (B), New York Botanical Garden (NY) y Tropicos.org del Missouri Botanical Garden. Se revisó la correcta determinación de los especímenes y se capturó la información correspondiente al acrónimo del herbario en el que se encontró depositado el material, el colector, número de recolecta, municipio, localidad, tipo de vegetación (sensu Rzedowski, 2006) y altitud. Para la presentación del listado se siguió la clasificación propuesta por Smith et al. (2006), incluyendo entre paréntesis las actualizaciones nomenclaturales que para algunos grupos se han publicado en trabajos posteriores (Ebihara et al., 2006; Moran et al., 2010; Labiak, 2011; Regalado y Prada 2011; Ø11gaard, 2012; Krömer et al., 2013a; Smith y Tejero-Díez, 2014). Los nombres de los taxa se cotejaron con The Plant List (2013) y The International Plant Names Index (2014). Es importante señalar que solo se consideró el material en cuyas etiquetas se indicaba de manera explícita el hábito epífito. En los casos para los que no encontramos ejemplares en las colecciones revisadas, se incluyó la referencia bibliográfica del trabajo en el que fueron citados. De manera paralela a la revisión bibliográfica y de herbario, se realizaron viajes de recolección a distintas zonas de la entidad con tipos de vegetación favorables para el desarrollo de epífitas, durante los cuales se recolectaron alrededor de 100 números, cuyo primer duplicado se depositó en el Herbario Metropolitano Ramón Riba y Nava Esparza (UAMIZ).

\section{RESULTADOS}

En total se revisaron 1800 ejemplares de helechos y licofitos epífitos de Veracruz que, después de cotejar su identidad taxonómica, corresponden a 11 familias, 33 géneros y 173 especies con 8 variedades y un híbrido (Apéndice 1), cifra que representa $30.6 \%$ de las 565 especies de pteridobiontes registrados por TejeroDíez et al. (2011) y Krömer et al. (2013a) para la entidad. Si además se suman los taxa mencionados en distintos trabajos (Lira y Riba, 1984; Vázquez-Torres et al., 2006; Pérez-Peña, 2007; Mehltreter, 2008; Viccon-Esquivel, 2009; Krömer et al., 
2013a, b; Carvajal-Hernández et al., 2014), para los que no encontramos ejemplares de respaldo en las colecciones revisadas (Apéndice 2), pero que con base en el trabajo de Mickel y Smith (2004) se sabe que pueden presentar el hábito epífito, el número se incrementa a 196 especies, lo que corresponde a $34.7 \%$ del total conocido. Cabe señalar que en las cifras dadas se consideraron las plantas holoepífitas, epífitas facultativas y hemiepífitas, pero no se incluyeron las epífitas ocasionales (Apéndice 3).

Las familias mejor representadas a nivel genérico son: Polypodiaceae con 14 (42.4\%), Pteridaceae con 5 (15.1\%), Dryopteridaceae con 4 (12.1\%) e Hymenophyllaceae y Lomariopsidaceae con 2 cada una (6\%) (Fig. 1). A nivel específico lo son: Polypodiaceae con 76 (43.9\%), Hymenophyllaceae con 28 (16.1\%), Dryopteridaceae con 21 (12.1\%), Aspleniaceae con 19 (11\%) y Pteridaceae con 10 (5.8\%) (Fig. 1). Los géneros con mayor número de especies son: Polypodium con 31 (17.9\%), Asplenium con 19 (11\%), Elaphoglossum con 16 (9.2\%), Trichomanes con 15 (8.7\%) e Hymenophyllum con 13 (7.5\%) (Fig. 2).

Del total de especies de pteridofitas epífitas presentes en la entidad, cinco se encuentran dentro de la Norma Oficial Mexicana 059 (SEMARNAT, 2010): Asplenium auritum, A. serratum, Huperzia dichotoma (=Phlegmariurus dichotomus), Psilotum complanatum y Serpocaulon triseriale, lo que representa $20.8 \%$ de las 24 especies que en esta categoría citaron Tejero-Díez et al. (2011). No se registran especies epífitas endémicas en el estado.

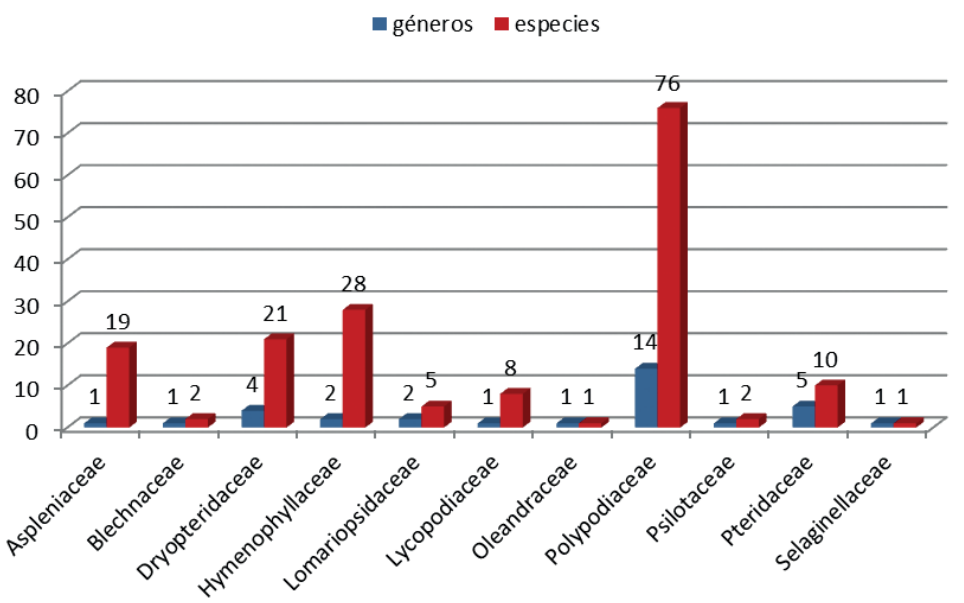

Fig. 1. Número de géneros y especies por familia en Veracruz. 


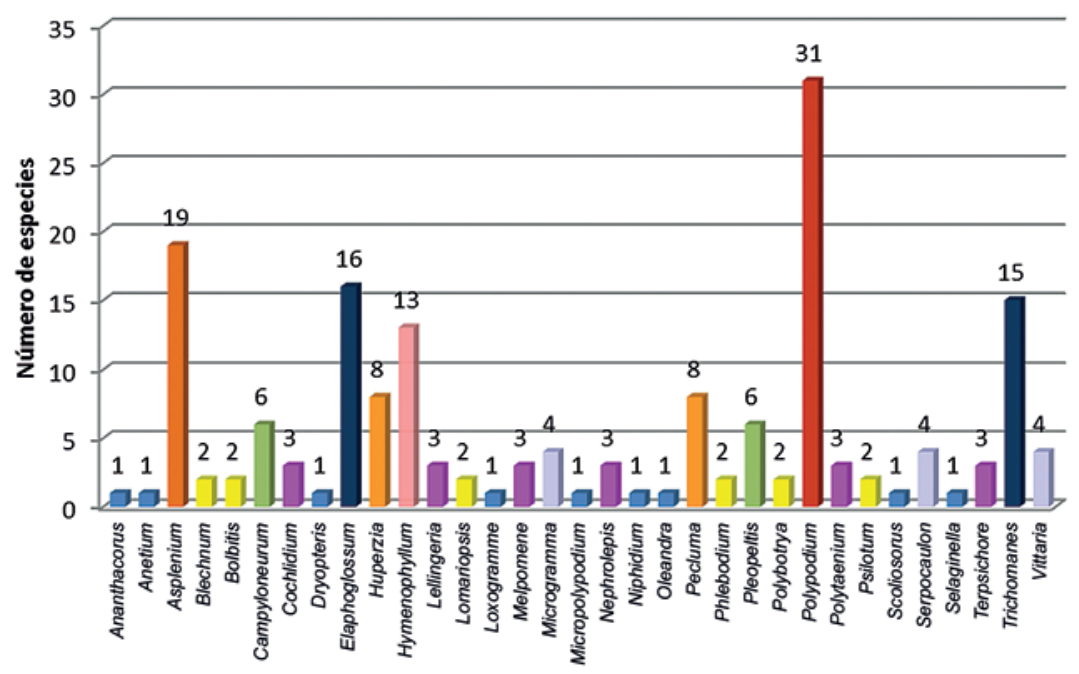

Fig. 2. Número de especies por género en Veracruz.

En lo que se refiere a la distribución de especies de helechos epífitos por tipo de vegetación (Fig. 3), los que presentan mayor número de taxa son el bosque mesófilo de montaña con 119, el bosque tropical perennifolio con 84 y el bosque de Quercus con 65, lo que constituye el 32.2\%, 28.4\% y 23.4\% respectivamente, del total de especies de pteridobiontes reportadas por Tejero-Díez et al. (2011) para cada una de estas asociaciones vegetales en el estado.

La mayoría de las especies crecen entre los 751 y 1750 m s.n.m. (Fig. 4), siendo Microgramma lycopodioides, M. nitida, Phlebodium decumanum y Vittaria lineata las que se ubican en la parte baja del intervalo altitudinal $(0 \mathrm{~m})$, mientras que Pleopeltis polylepis se presenta a mayor altitud (3200 m).

De los 212 municipios en que se divide el estado, en 113 (53.3\%) hay al menos un registro del grupo en estudio y en 99 ninguno. Los que presentan el mayor número de especies son: San Andrés Tuxtla con 73, Soteapan con 62, Atzalan con 52, Catemaco con 50, Acajete con 39, Huatusco con 36, Hidalgotitlán con 33, Zongolica con 33, Tlalnelhuayocan con 31, Xalapa con 28 y Xico con 24 (Fig. 5).

\section{DISCUSIÓN Y CONCLUSIÓN}

La comparación de nuestros resultados con los obtenidos en trabajos que cuentan con información a nivel estatal o regional sobre el tema (Cuadro 1) pone de 


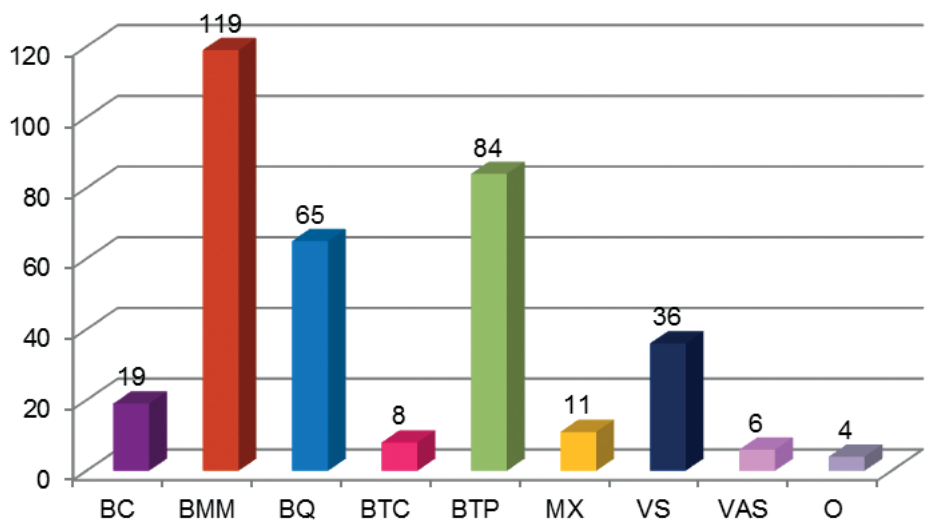

Fig. 3. Número de especies por tipo de vegetación en Veracruz. ( $\mathrm{BC}=$ bosque de coníferas; $\mathrm{BMM}=$ bosque mesófilo de montaña; $\mathrm{BQ}=$ bosque de Quercus; $\mathrm{BTC}=$ bosque tropical caducifolio; $\mathrm{BTP}=$ bosque tropical perennifolio; $\mathrm{MX}=$ matorral xerófilo; $\mathrm{VS}=$ vegetación secundaria; VAS = vegetación acuática y subacuática; $\mathrm{O}=$ otros).

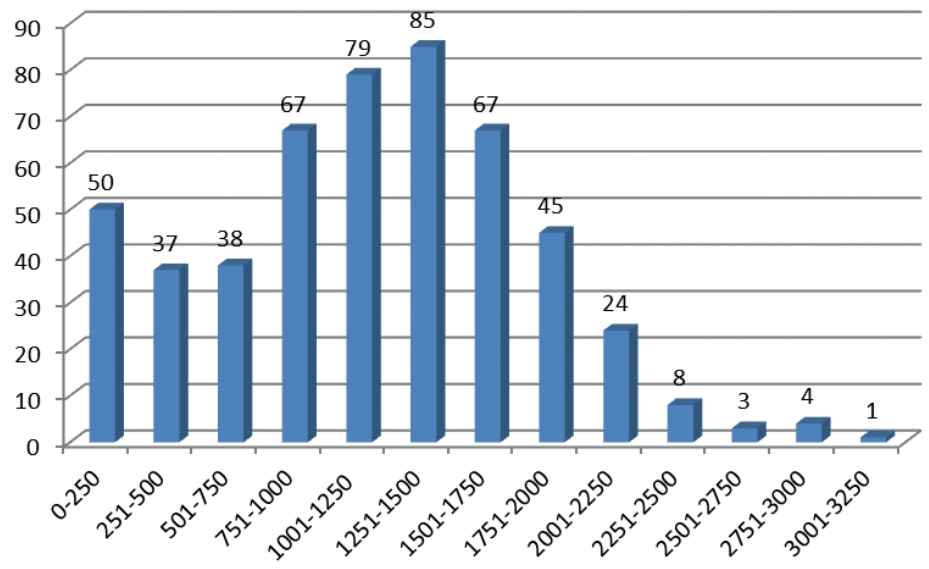

Fig. 4. Número de especies por intervalo altitudinal en Veracruz.

manifiesto la importancia del estado de Veracruz en lo que se refiere a riqueza de especies de pteridofitas y licofitas epífitas, al contar en su territorio con entre 173 y 196 taxa (esta última cifra si se consideran los citados en la literatura), quedando solo por debajo de Chiapas y Oaxaca, que cuentan con 244 y 201 especies con esta 
Mendoza-Ruiz et al.: Helechos y licofitos epífitos de Veracruz, México

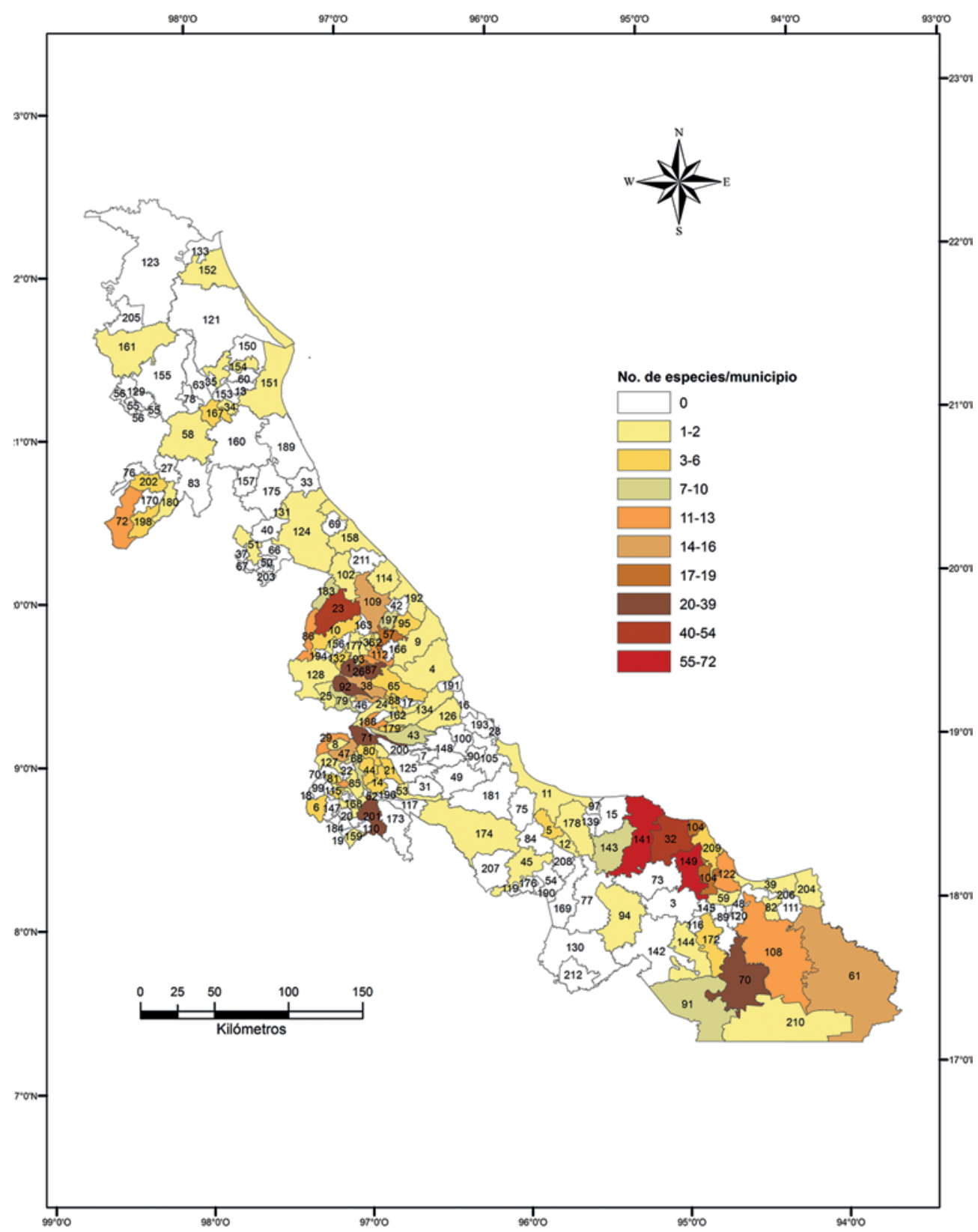

Fig. 5. Número de especies de helechos y licofitos por municipio en Veracruz. 
Cuadro 1. Número de especies de helechos y licofitos epífitos en Veracruz y otros estados y regiones de México.

\begin{tabular}{lcc}
\hline Estado/ región/ país & $\begin{array}{c}\text { Extensión } \\
\left(\mathrm{km}^{2}\right)\end{array}$ & $\begin{array}{c}\text { Núm. especies } \\
\text { epífitas }\end{array}$ \\
\hline Veracruz (en este estudio) & 71820 & $\begin{array}{c}173+23 \\
\text { citadas (196) }\end{array}$ \\
Veracruz (Tejero-Díez et al. 2011) & 71820 & 168 \\
Bajío y regiones adyacentes (Ceja-Romero et al., 2012) & 50000 & 51 \\
Chiapas (Wolf y Flamenco-Sandoval, 2005) & 73311 & 244 \\
Estado de México (Tejero-Díez y Arreguín Sánchez, 2004) & 23244 & 27 \\
Hidalgo (Ceja-Romero et al., 2010) & 20905 & 66 \\
Oaxaca (Mickel y Beitel, 1988) & 93757 & 191 \\
Oaxaca (Mickel y Smith, 2004) & 93757 & 201 \\
México (Mickel y Smith, 2004) & 1964375 & 265 \\
\hline
\end{tabular}

forma de vida respectivamente (Wolf y Flamenco-Sandoval, 2003; Mickel y Smith, 2004). También resulta evidente que a medida que se tiene un mejor conocimiento florístico de las diferentes regiones de la entidad, se incrementa de manera gradual el número de taxa epífitos reportados en el estado (Cuadro 2).

En lo concerniente a la distribución de epífitas por tipo de vegetación (sensu Rzedowski, 2006), encontramos que de manera similar a lo que ocurre en entidades como Hidalgo y Chiapas (Wolf y Flamenco-Sandoval, 2003; Ceja-Romero et al., 2010), y a pesar de que en Veracruz el bosque mesófilo de montaña ha sido transformado en un $90 \%$ de su superficie original para otros usos (Williams-Linera, 2002), es la comunidad que cuenta con el mayor número de taxa con esta forma de vida, representando $18.8 \%$ de las 630 especies de helechos reportadas en el país para este tipo de vegetación por Tejero-Díez et al. (2014). Lo anterior corrobora una vez más que no obstante ocupar una extensión reducida, es la asociación vegetal en la que se encuentran más diversificadas las plantas epífitas (Rzedowski, 1996, 2006). En este sentido es importante tomar en cuenta que seis de las 15 subregiones de bosque mesófilo de montaña, consideradas por la CONABIO (2010) como de prioridad crítica, están o incluyen alguna porción del estado (Fig. 6). Además algunos de los municipios que se localizan dentro de ellas como Huayacocotla, Llamatlán, Tlaltetela, Texcatepec, Zontecomatlán, entre otros, registran en este trabajo un número de especies bajo o nulo (Fig. 5), situación que podría cambiar una vez que se cuente con un mejor conocimiento florístico de ellos. 
Cuadro 2. Número de especies de helechos y licofitos epífitos registrados para el estado de Veracruz.

\begin{tabular}{|c|c|c|c|c|c|c|}
\hline \multirow[b]{2}{*}{ Familia y Géneros } & \multicolumn{2}{|c|}{ Este trabajo } & \multicolumn{2}{|c|}{ Tejero-Díez et al. 2011} & \multicolumn{2}{|c|}{ Mickel y Smith, 2004} \\
\hline & géneros & especies & géneros & especies & géneros & especies \\
\hline Aspleniaceae & 1 & 19 & 2 & 16 & 2 & 17 \\
\hline Asplenium & & 19 & & 15 & & 16 \\
\hline $\begin{array}{c}\text { Loxoscaphe } \\
\text { (dudoso) }\end{array}$ & & 0 & & 1 & & 1 \\
\hline Holodictyum & & 0 & & 0 & & 0 \\
\hline $\begin{array}{l}\text { Blechnaceae } \\
\text { Blechnum }\end{array}$ & 1 & 2 & 1 & 2 & 1 & 3 \\
\hline Dryopteridaceae & 4 & 21 & 3 & 22 & 2 & 18 \\
\hline Bolbitis & & 2 & & 1 & & 1 \\
\hline Dryopteris & & 1 & & 0 & & 0 \\
\hline Elaphoglossum & & 16 & & 20 & & 17 \\
\hline Polybotrya & & 2 & & 1 & & 0 \\
\hline Hymenophyllaceae & 2 & 28 & 2 & 31 & 2 & 26 \\
\hline Hymenophyllum & & 13 & & 14 & & 13 \\
\hline Trichomanes & & 15 & & 17 & & 13 \\
\hline Lomariopsidaceae & 2 & 5 & 2 & 5 & 2 & 6 \\
\hline Lomariopsis & & 2 & & 2 & & 2 \\
\hline Nephrolepis & & 3 & & 3 & & 4 \\
\hline $\begin{array}{l}\text { Lycopodiaceae } \\
\text { Huperzia }\end{array}$ & 1 & 8 & 1 & 8 & 1 & 8 \\
\hline $\begin{array}{l}\text { Oleandraceae } \\
\text { Oleandra }\end{array}$ & 1 & 1 & 1 & 1 & 1 & 1 \\
\hline Polypodiaceae & 14 & 76 & 14 & 71 & 13 & 65 \\
\hline Campyloneurum & & 6 & & 8 & & 7 \\
\hline Cochlidium & & 3 & & 2 & & 2 \\
\hline Lellingeria & & 3 & & 2 & & 2 \\
\hline Loxogramme & & 1 & & 1 & & 1 \\
\hline Melpomene & & 3 & & 3 & & 3 \\
\hline Microgramma & & 4 & & 3 & & 3 \\
\hline Micropolypodium & & 1 & & 2 & & 2 \\
\hline Niphidium & & 1 & & 1 & & 1 \\
\hline Pecluma & & 8 & & 7 & & 4 \\
\hline Phlebodium & & 2 & & 2 & & 2 \\
\hline Pleopeltis & & 6 & & 7 & & 7 \\
\hline Polypodium & & 31 & & 27 & & 27 \\
\hline Serpocaulon & & 4 & & 2 & & 0 \\
\hline Terpsichore & & 3 & & 4 & & 4 \\
\hline
\end{tabular}


Cuadro 2. Continuación.

\begin{tabular}{|c|c|c|c|c|c|c|}
\hline \multirow[b]{2}{*}{ Familia y Géneros } & \multicolumn{2}{|c|}{ Este trabajo } & \multicolumn{2}{|c|}{ Tejero-Díez et al. 2011} & \multicolumn{2}{|c|}{ Mickel y Smith, 2004} \\
\hline & géneros & especies & géneros & especies & géneros & especies \\
\hline $\begin{array}{l}\text { Psilotaceae } \\
\text { Psilotum }\end{array}$ & 1 & 2 & 1 & 1 & 1 & 1 \\
\hline Pteridaceae & 5 & 10 & 5 & 10 & 6 & 11 \\
\hline Ananthacorus & & 1 & & 1 & & 1 \\
\hline Anetium & & 1 & & 1 & & 1 \\
\hline Polytaenium & & 3 & & 3 & & 3 \\
\hline Radiovittaria & & 0 & & 0 & & 1 \\
\hline Scoliosorus & & 1 & & 1 & & 1 \\
\hline Vittaria & & 4 & & 4 & & 4 \\
\hline Selaginellaceae & 1 & 1 & 1 & 1 & 0 & 0 \\
\hline Total & 33 & 173 & 33 & 168 & 31 & 156 \\
\hline
\end{tabular}

El bosque tropical perennifolio, segundo lugar en riqueza de taxa epífitos, cuenta con su mejor representación en la región de Los Tuxtlas (Castillo-Campos et al., 2011), zona cuya relevancia se puede apreciar, al observar que tres de los cinco municipios con mayor número de especies, San Andrés Tuxtla, Catemaco y Soteapan, forman parte de ella (Fig. 7). Es interesante notar que para Tatahuicapan de Juárez, que también es parte de la zona, se tuvo un número bajo de registros, lo que podría indicar la necesidad de más trabajo de colecta en el lugar. Otra de las regiones importantes para este tipo de vegetación es la de Uxpanapa, considerada como un área de alta biodiversidad con presencia de endemismos atribuidos a los cambios climáticos ocurridos en el Cenozoico (Wendt, 1989), cuya superficie está conformada por los municipios de Hidalgotitlán, uno de los que tiene mayor riqueza específica en este trabajo, Jesús Carranza, Las Choapas, Minatitlán y Uxpanapa (Fig. 6) y para la que por sus características, Riba y Pérez García (1979) señalaron la posibilidad de encontrar más especies de helechos, sobre todo hacia la llamada Sierra de Tres Picos.

En cuanto a los bosques de Quercus, resulta interesante notar que, no obstante que se ha señalado que en la entidad prácticamente no existen fragmentos de ellos en buen estado de conservación, ni tampoco áreas protegidas que los incluyan (Castillo-Campos et al., 2011), ocupan el tercer lugar en número de plantas epífitas, lo que pone de manifiesto la importancia de su estudio. 
Mendoza-Ruiz et al.: Helechos y licofitos epífitos de Veracruz, México

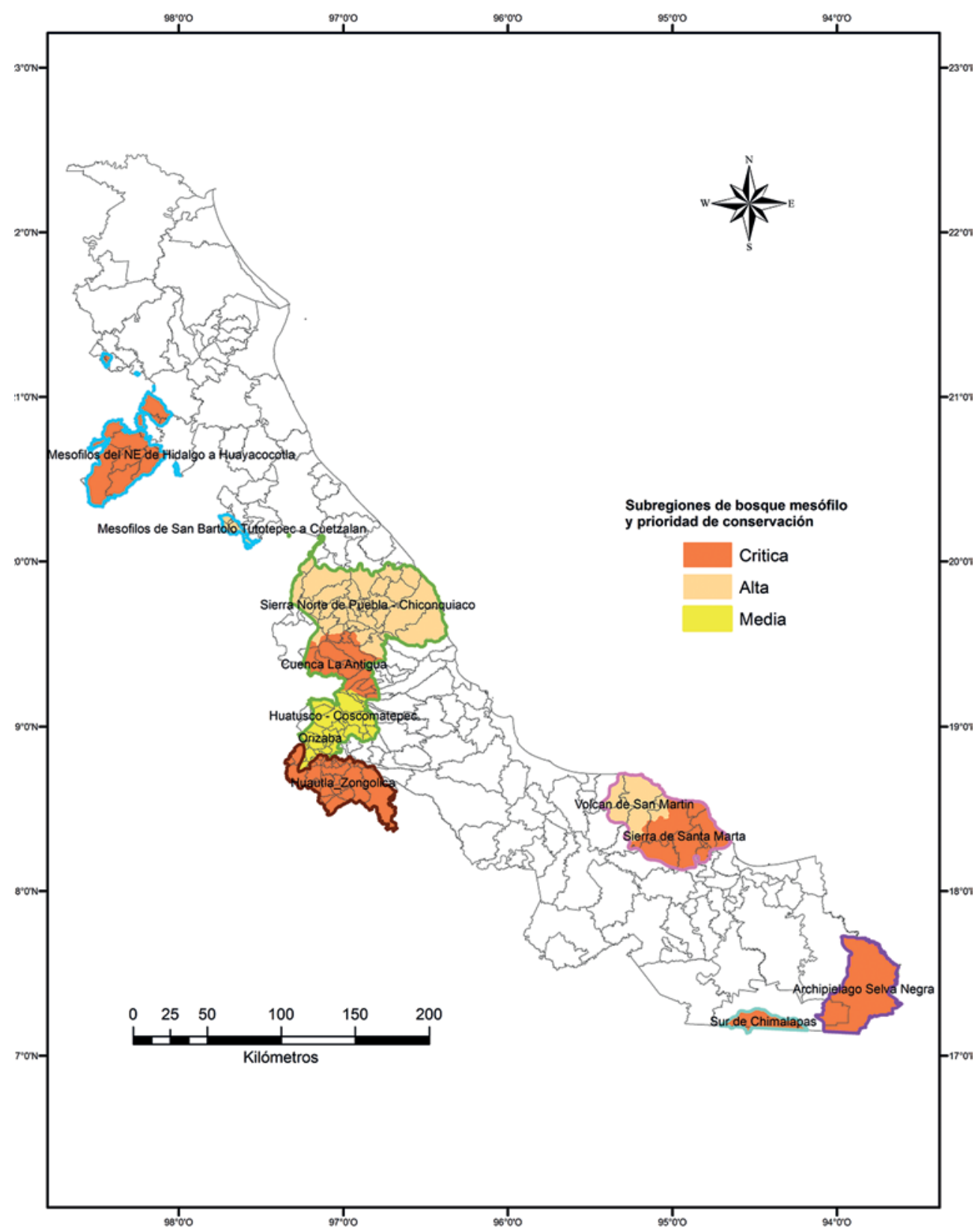

Fig. 6. Subregiones y categorías de prioridad para la conservación del bosque mesófilo de montaña en Veracruz. 


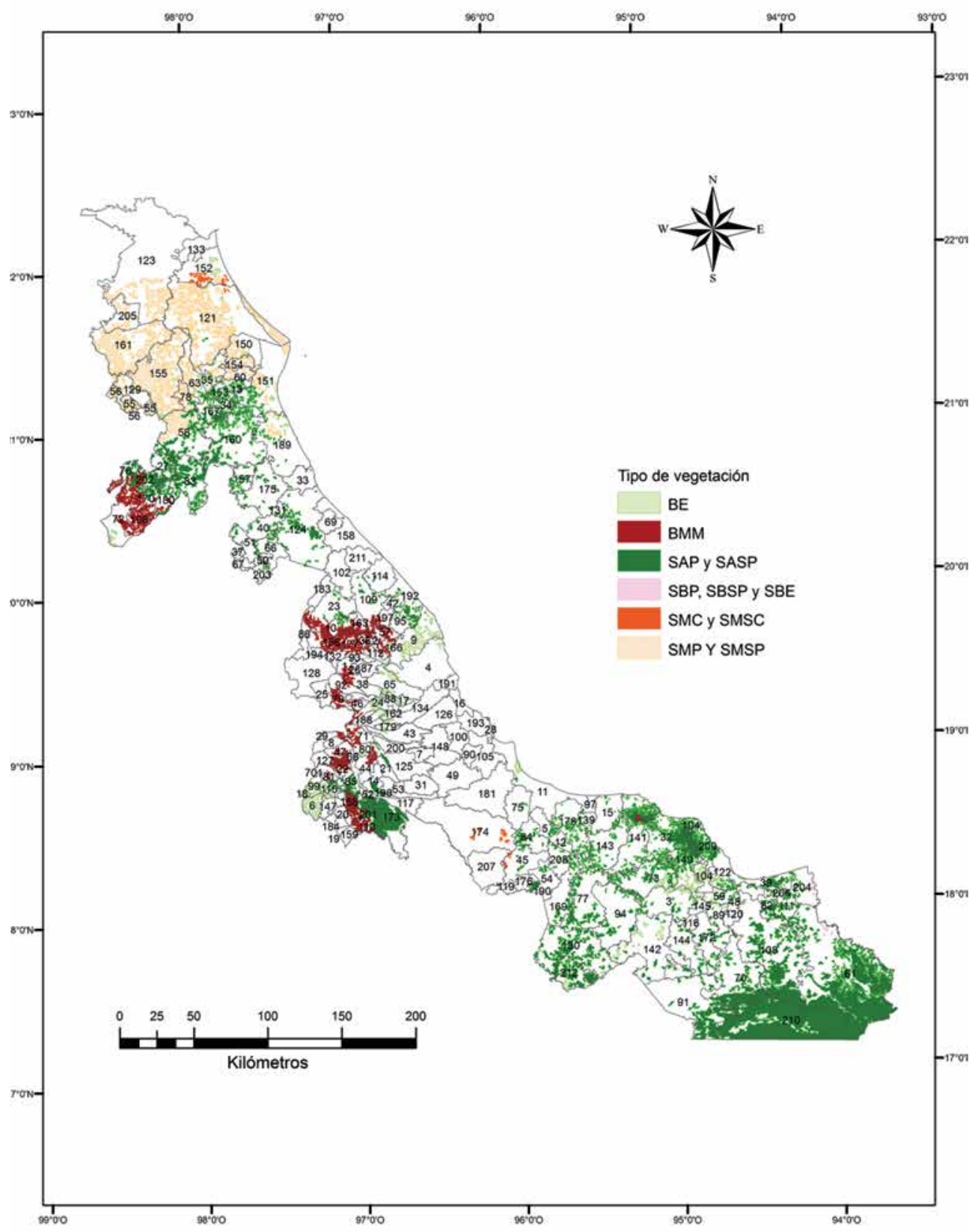

Fig. 7. Principales tipos de vegetación favorables para el desarrollo de las epífitas en Veracruz. (Fuente: modificado a partir de CONABIO, 1999). 
Con respecto al intervalo altitudinal y su relación con la riqueza de especies, de manera similar a lo reportado en diversos estudios sobre pteridofitas (Krömer et al., 2005; Krömer et al., 2013b; Salazar et al., 2013), se puede observar una curva en forma de campana con el valor máximo entre los 1251 y $1500 \mathrm{~m}$, disminuyendo de manera gradual hacia los extremos (Fig. 4). Llama la atención el número de especies presentes entre los 0 y los $750 \mathrm{~m}$, sobre todo si comparamos con lo registrado para Hidalgo y el área del Bajío y regiones adyacentes (Ceja-Romero et al., 2010, 2012), lo que se puede relacionar con la presencia del bosque tropical perennifolio en el estado, tipo de vegetación prácticamente ausente en la zona del Bajío y restringido a la zona limítrofe con Veracruz y San Luis Potosí en Hidalgo (INEGI, 1992).

Finalmente, se puede observar que, a pesar de que ya se cuenta con una buena cantidad de información sobre las pteridofitas epífitas de Veracruz, aún hay municipios que no obstante ser considerados dentro de los más diversos del estado, como por ejemplo Perote, Tezonapa y Tatatila (Márquez-Ramírez y Márquez-Ramírez, 2009), y presentar los tipos de vegetación favorables para el desarrollo de las plantas con esta forma de vida, sólo tienen un registro o ninguno. Esta situación indica la necesidad de llevar a cabo proyectos de exploración, con el fin de determinar si esto se debe al deterioro del ecosistema en cuestión o a que aún no se ha realizado trabajo de recolección en la zona. Sin embargo, independientemente de ello, es incuestionable la importancia que tiene la entidad en lo que a biodiversidad se refiere.

\section{AGRADECIMIENTOS}

A Thorsten Krömer por enriquecer el trabajo con sus valiosos comentarios y sugerencias. A los revisores anónimos que contribuyeron con sus observaciones a mejorar el manuscrito. A Zoraya Ceja por su asesoría en la elaboración de los mapas. A los curadores de los herbarios CORU, ENCB, FCME, IEB, MEXU, OAX, UAMIZ, XAL y XALU por las facilidades proporcionadas para la consulta de sus colecciones.

\section{LITERATURA CITADA}

Acosta-Rosado, I. 2002. Vegetación y flora del municipio de Xico, Veracruz, México. Tesis de licenciatura. Facultad de Biología, Universidad Veracruzana. Xalapa, México. $150 \mathrm{pp}$. 
Alanís-Méndez, J. L., F. M. Muñoz Arteaga, M. López Ortega, L. Cuervo López, y B. E. Raya Cruz. 2007. Aportes al conocimiento de las epífitas (Bromeliaceae, Cactaceae y Orchidaceae) en dos tipos de vegetación del municipio de Pánuco, Veracruz, México. Revista UDO Agricola 7(1): 160-174.

Benzing, D. 1990. Vascular epiphytes. General biology and related biota. Cambridge University Press. Cambridge, UK. 353 pp.

Bongers, F., J. Pompa, J. Meave del Castillo y J. Carabias. 1988. Structure and floristic composition of the lowland rain forest of Los Tuxtlas, Mexico. Vegetatio 74: 55-80.

Carreño-Rocabado, I. G. 2006. Evaluación de los cafetales bajo sombra y fragmentos de bosque adyacentes como hábitats para conservar la diversidad de los helechos en el estado de Veracruz, México. Tesis de maestría. Ecología y Recursos Naturales, Instituto de Ecología, A.C., Xalapa, México. 120 pp.

Carvajal-Hernández C., T. Krömer y M. Vázquez-Torres. 2014. Riqueza y composición florística de pteridobiontes en bosque mesófilo de montaña y ambientes asociados, en el centro de Veracruz, México. Rev. Mex. Biodiv. 85: 491-501.

Castillo-Campos G., R. Robles-Gonzales y M. E. Medina-Abreo. 2003. Flora y vegetación de la Sierra Cruz Tetela, Veracruz, México. Polibotánica 15: 41-87.

Castillo-Campos, G. y V. E. Luna Monterrojo. 2009. Flora y vegetación del municipio de Coatepec, Veracruz. Flora de Veracruz 1: 1-288.

Castillo-Campos, G., S. Avendaño-Reyes y M. E. Medina-Abreo. 2011. Flora y Vegetación. In: Cruz-Angón, A. (ed.). La biodiversidad en Veracruz: Estudio de estado. Vol. I. Comisión Nacional para el Conocimiento y Uso de la Biodiversidad, Gobierno del Estado de Veracruz, Universidad Veracruzana, Instituto de Ecología, A.C., México, D.F., México. pp. 163-179.

Ceja-Romero, J., A. Mendoza-Ruiz, A. R. López-Ferrari, A. Espejo, B. Pérez-García y J. García Cruz. 2010. Las epífitas vasculares del estado de Hidalgo, México: diversidad y distribución. Acta Bot. Mex. 93: 1-39.

Ceja-Romero, J., A. Espejo-Serna, J. García-Cruz, A. R. López-Ferrari, A. Mendoza-Ruiz y B. Pérez-García. 2012. Epífitas vasculares del Bajío y de regiones adyacentes. Flora del Bajío y de Regiones Adyacentes. Fascículo complementario XXVIII: 1-55.

Cortés Flores, I. S. 2009. Las bromeliáceas en relictos de vegetación en la propuesta para el establecimiento de reserva de la biósfera cuencas de los ríos Nautla, Misantla y Colipa, Veracruz. Tesis de licenciatura. Facultad de Biología, Universidad Veracruzana. Xalapa, México. 152 pp.

CONABIO. 1999. Uso de suelo y vegetación modificado por CONABIO. Mapa. Escala 1: 1000 000. Comisión Nacional para el Conocimiento y Uso de la Biodiversidad. México, D.F., México.

CONABIO. 2010. El bosque mesófilo de montaña en México: Amenazas y oportunidades para su conservación y manejo sostenible. Comisión Nacional para el Conocimiento y Uso de la Biodiversidad. México D.F., México. 197 pp.

Dubuisson J. Y., H. Schneider y S. Hennequin. 2009. Epiphytism in ferns: diversity and history. Comp. Rend. Biol. 332: 120-128.

Ebihara, A., J. Y. Dubuisson, K. Iwatsuki, S. Hennequin y M. Ito. 2006. A taxonomic revision of Hymenophyllaceae. Blumea 51: 221-280. 
Espejo-Serna, M. A. y A. R. López-Ferrari. 2011. Diversidad y distribución de las Bromeliáceas. In: Cruz-Angón, A. (ed.). La biodiversidad en Veracruz: Estudio de estado. Vol. II. Comisión Nacional para el Conocimiento y Uso de la Biodiversidad, Gobierno del Estado de Veracruz, Universidad Veracruzana, Instituto de Ecología, A.C. México, D.F., México. pp. 177-189.

Flores-Méndez, H. 2008. Epífitas vasculares del bosque mesófilo de montaña de la comunidad La Quinta, Zongolica, Veracruz. Tesis de licenciatura. Facultad de Ciencias Biológicas y Agropecuarias, Zona Orizaba-Córdoba, Universidad Veracruzana. Córdoba, México. 125 pp.

Flores-Palacios, A. 2003. El efecto de la fragmentación del bosque mesófilo en la comunidad de plantas epífitas vasculares. Tesis de doctorado. Instituto de Ecología A. C., Xalapa, México. 159 pp.

Flores-Palacios, A. y J. G. García-Franco. 2001. Sampling methods for vascular epiphytes: their effectiveness in recording species richness and frequency. Selbyana 22(2): 181191.

Flores-Palacios, A. y J. G. García-Franco. 2004. Effect of isolation on the structure and nutrient content of oak epiphyte communities. Pl. Ecol. 173: 259-269.

Flores-Palacios, A. y J. G. García-Franco. 2006. The relationship between tree size and epiphyte species richness: testing four different hypotheses. J. Biogeogr. 33: 323-330.

Flores-Palacios, A. y J. G. García-Franco. 2008. Habitat isolation changes the beta diversity of the vascular epiphyte community in lower montane forest, Veracruz, Mexico. Biodiv. Conserv. 17: 191-207.

Flores-Palacios, A. y S. Valencia-Díaz. 2007. Local illegal trade reveals unknown diversity and involves a high species richness of wild vascular epiphytes. Biol. Conserv. 136: 372-387.

Flores-Palacios, A., J. G. García-Franco, S. Valencia-Díaz, L. Solís-Montero y A. CruzAngón. 2011. Diversidad y conservación de plantas epífitas vasculares en el centro del estado. In: Cruz-Angón, A. (ed.). La biodiversidad en Veracruz: Estudio de estado. Vol. I. Comisión Nacional para el Conocimiento y Uso de la Biodiversidad, Gobierno del Estado de Veracruz, Universidad Veracruzana, Instituto de Ecología, A. C. México, D.F., México. pp. 493-501.

García-Cruz, J. y L. Sánchez-Saldaña. 1999. Orchidaceae II. Epidendrum 1. Flora de Veracruz 112: 1-110.

García-Franco, J. G. 1996. Distribución de epífitas vasculares en matorrales costeros de Veracruz. Acta Bot. Mex. 37: 1-9.

García-Franco, J. G. y T. Toledo-Aceves. 2008. Cap. 5 Epífitas vasculares: bromelias y orquídeas. In: Manson, R. H., V. Hernández-Ortiz, S. Gallina y K. Mehltreter (eds.). Agroecosistemas cafetaleros de Veracruz: biodiversidad, manejo y conservación. Instituto Nacional de Ecología. México, D.F., México. pp. 69-82.

García-Franco, J. G., G. Castillo-Campos, K. Mehltreter, M. L. Martínez y G. Vázquez. 2008. Composición florística de un bosque mesófilo del centro de Veracruz, México. Bol. Soc. Bot. Méx. 83: 37-52.

Gómez-Díaz, J. A. 2010. Comparación florística de epífitas vasculares entre un bosque mesófilo de montaña y un acahual en el municipio de Tlalnelhuayocan, Veracruz. 
Tesis de licenciatura. Facultad de Biología, Universidad Veracruzana. Xalapa, México. $111 \mathrm{pp}$.

Gómez-Ortega, V. A. 2010. Contribución al conocimiento de la vegetación y flora del municipio de Acatlán, Veracruz, Tesis de licenciatura. Universidad Veracruzana, Facultad de Biología, Xalapa, México. 56 pp.

Gómez-Pompa, A y G. Castillo-Campos. 2010. La vegetación de Veracruz. In: GómezPompa, A., T. Krömer y R. Castro-Cortés (coords.). Atlas de la flora de Veracruz. Un patrimonio natural en peligro. Gobierno del estado de Veracruz-Universidad Veracruzana. Xalapa, México. pp. 57-76.

Gutiérrez-García, G. y M. Ricker. 2011. Climate and climate change in the region of Los Tuxtlas (Veracruz, Mexico): A statistical analysis. Atmósfera 24(4): 347-373.

Hietz, P. y U. Hietz-Seifert. 1995. Composition and ecology of vascular epiphyte communities along an altitudinal gradient in central Veracruz, Mexico. J. Veg. Sci. 6: 487-498.

Hietz, P. y O. Briones. 1998. Correlation between water relations and within-canopy distribution of epiphytic ferns in a Mexican cloud forest. Oecologia 114(3): 305316.

Hietz, P. y O. Briones. 2004. Adaptaciones y bases fisiológicas de la distribución de los helechos epífitos en un bosque de niebla. In: Marino-Cabrera, H. (ed.). Fisiología ecológica en plantas mecanismos y respuestas a estrés en los ecosistemas. Ediciones Universitarias de Valparaíso. Valparaíso, Chile. pp. 121-138.

Hietz, P. 2005. Conservation of vascular epiphyte diversity in Mexican coffee plantations. Conserv. Biol. 19(2): 391-399.

INEGI. 1992. Síntesis geográfica del estado de Hidalgo. Instituto Nacional de Estadística, Geografía e Informática. Aguascalientes, México. 134 pp.

INEGI. 2012a. Anuario estadístico y geográfico de Veracruz de Ignacio de la Llave 2012. Instituto Nacional de Estadística y Geografía. Aguascalientes, México. 1077 pp.

INEGI. 2012b. Perspectiva estadística de Veracruz 2012. Instituto Nacional de Estadística y Geografía. Aguascalientes, México. 103 pp.

INEGI. 2013. Anuario estadístico y geográfico de Veracruz de Ignacio de la Llave. Instituto Nacional de Estadística y Geografía. Aguascalientes, México. 1067 pp.

Kress, W. J. 1986. The systematic distribution of vascular epiphytes: an update. Selbyana 9(1): 2-22.

Kress, W. J. 1989. The systematic distribution of vascular epiphytes. In: Lüttge, U. (ed.). Vascular plants as epiphytes: evolution and ecophysiology. Springer-Verlag, Incorporated. New York, USA. pp. 234-261.

Krömer, T., M. Kessler, S. R. Gradstein y A. Acebey. 2005. Diversity patterns of vascular epiphytes along an elevational gradient in the Andes. J. Biogeogr. 32: 1799-1809.

Krömer, T., A. Acebey y A. R. Smith. 2013a. Taxonomic update, distribution and conservation status of grammitid ferns (Polypodiaceae, Polypodiopsida) in Veracruz State, Mexico. Phytotaxa 82(1): 29-84.

Krömer T., A. R. Acebey, J. Kluge y M. Kessler. 2013b. Effects of altitude and climate in determining elevational plant species richness patterns: a case study from Los Tuxtlas, Mexico. Flora 208: 197-210. 
Labiak, P. H. 2011. Stenogrammitis, a new genus of grammitid fern segregated from Lellingeria (Polypodiaceae). Brittonia 63(1):139-149.

Lira, R. 1983. Contribución al conocimiento de la flora pteridológica de la Sierra de Santa Marta, Los Tuxtlas, Veracruz. Tesis de licenciatura, Facultad de Ciencias, Universidad Nacional Autónoma de México. México, D.F., México. 133 pp.

Lira, R. y R. Riba. 1984. Aspectos fitogeográficos y ecológicos de la flora Pteridofítica de la sierra de Santa Marta, Veracruz, México. Biótica 9(4): 451-467.

Luna, I., L. Almeida, L. Villers y L. Lorenzo. 1988. Reconocimiento florístico y consideraciones fitogeográficas del bosque mesófilo de montaña de Teocelo, Veracruz. Bol. Soc. Bot. Méx. 48: 35-63.

Márquez-Ramírez, W. y J. Márquez-Ramírez. 2009. Municipios con mayor biodiversidad en Veracruz. Foresta Veracruzana 11(2): 43-50.

Mehltreter, K. 2008. Helechos. In: Manson, R. H., V. Hernández-Ortiz, S. Gallina y K. Mehltreter (eds.). Agroecosistemas cafetaleros de Veracruz: Biodiversidad, Manejo y Conservación. Instituto Nacional de Ecología (INE), México, D.F., México. pp. 83-93.

Mehltreter, K., A. Flores-Palacios y J. G. García-Franco. 2005. Host preferences of lowtrunk vascular epiphytes in a cloud forest of Veracruz, Mexico. J. Trop. Ecol. 21(6): 651-660.

Mickel, J. T. y J. M. Beitel. 1988. Pteridophyte flora of Oaxaca, Mexico. Mem. New York Bot. Gard. 46: 1-568.

Mickel, J. T. y A. R. Smith. 2004. The pteridophytes of Mexico. Mem. New York Bot. Gard. 88: 1-1054.

Morales-Linares, J. 2009. Diversidad de orquídeas en cuatro ambientes del ejido Rancho Viejo-Palmarejo, municipio de Emiliano Zapata, Veracruz. Tesis de licenciatura. Facultad de Biología, Universidad Veracruzana. Xalapa, México. 95 pp.

Moran, R. C., P. H. Labiak y M. Sundue. 2010. Synopsis of Mickelia, a newly recognized genus of bolbitidoid ferns (Dryopteridaceae). Brittonia 62: 337-356.

Øllgaard, B. 2012. New combinations in neotropical Lycopodiaceae. Phytotaxa 57: 10-22.

Palacios-Ríos, M. 1992. Las pteridofitas del estado de Veracruz. Tesis de maestría. Facultad de Ciencias, Universidad Nacional Autónoma de México. México, D.F., México. 364 pp.

Pérez-Maqueo, O., L. Muñoz-Villers, G. Vázquez. 2011. Hidrología. In: Cruz-Angón, A. (ed.). La biodiversidad en Veracruz. Estudio de estado. Vol. 1. Comisión Nacional para el Conocimiento y Uso de la Biodiversidad, Gobierno del Estado de Veracruz, Universidad Veracruzana, Instituto de Ecología, A.C. México, D.F., México. pp. 289292.

Pérez-Peña, A. 2007. Composición florística y diversidad vegetal de epífitas vasculares en tres tipos de vegetación en terrenos cercanos a la Estación de Biología Tropical Los Tuxtlas, Veracruz. Tesis de licenciatura. Facultad de Ciencias Biológicas y Agropecuarias zona Orizaba-Córdoba. Universidad Veracruzana. Córdoba, México. 58 pp.

Regalado, L. R. y C. Prada. 2011. The genus Hymenasplenium (Aspleniaceae) in Cuba, including new combinations for the neotropical species. Amer. Fern J. 101: 265-281. 
Riba, R. y B. Pérez-García. 1979. Estudio botánico y ecológico de la región del río Uxpanapa, Veracruz No. 9. Pteridofitas. Biótica 4(3): 135-139.

Rojas-Santiago, B. B. 2012. Restauración de la diversidad epifítica de helechos en un proyecto de reforestación en Huatusco, Veracruz. Tesis de licenciatura. Facultad de Ciencias Biológicas y Agropecuarias Zona: Orizaba-Córdoba, Universidad Veracruzana. Córdoba, México. 58 pp.

Rzedowski, J. 1996. Análisis preliminar de la flora vascular de los bosques mesófilos de montaña de México. Acta Bot. Mex. 35: 25-44.

Rzedowski, J., 2006. Vegetación de México. Comisión Nacional para el Conocimiento y Uso de la Biodiversidad. Edición digital. México, D.F., México. 504 pp.

Salazar-Rodríguez, J. L. 2010. Holoepífitas vasculares del bosque mesófilo de montaña del municipio de Acajete, Veracruz. Tesis de licenciatura. Facultad de Biología, Universidad Veracruzana. Xalapa, México. 42 pp.

Salazar, L., J. Homeier, M. Kessler, S. Abrahamczyk, M. Lehnert, T. Krömer y J. Kluge. 2013. Diversity patterns of ferns along elevation in Andean tropical forests. Plant Ecology and Diversity 8. Doi:10.1080/17550874.2013.843036

SEMARNAT. 2010. Norma Oficial Mexicana NOM-059-SEMARNAT-2010. Protección ambiental-Especies nativas de México de flora y fauna silvestres-Categorías de riesgo y especificaciones para su inclusión, exclusión o cambio-Lista de especies en riesgo. Diario Oficial de la Federación 30 de diciembre de 2010, México D.F. Disponible en: http://www.semarnat.gob.mx/temas/gestionambiental/vidasilvestre/Documents/ NOM_059_SEMARNAT_2010.pdf. Consultado en octubre 2012.

Smith, A. R., K. M. Pryer, E. Schuettpelz, P. Korall, H. Schneider y P. G. Wolf. 2006. A classification for extant ferns. Taxon 55(3): 705-731.

Smith A. R. y J. D. Tejero-Díez. 2014. Pleopeltis (Polypodiaceae), a redefinition of the genus and nomenclatural novelties. Botanical Sciences 92: 43-58.

Soto-Esparza, M. y L. E. Giddings-Berger. 2011. Clima. In: Cruz-Angón, A. (ed.). La biodiversidad en Veracruz: Estudio de estado. Vol. 1. Comisión Nacional para el Conocimiento y Uso de la Biodiversidad, Gobierno del Estado de Veracruz, Universidad Veracruzana, Instituto de Ecología, A.C. México. pp. 35-52.

Tejero-Díez, J. D. y M. L. Arreguín-Sánchez. 2004. Lista con anotaciones de los pteridófitos del Estado de México, México. Acta Bot. Mex. 69: 1-82.

Tejero-Díez, D., A. Torres-Díaz, J. T. Mickel, K. Mehltreter y T. Crémor. 2011. Helechos y licopodios. In: Cruz-Angón, A. (ed.). La Biodiversidad en Veracruz: Estudio de estado. Vol. II. Comisión Nacional para el Uso de la Biodiversidad, Gobierno del Estado de Veracruz, Universidad Veracruzana, Instituto de Ecología, A.C. México, D.F., México. pp. 97-114.

Tejero-Díez, D., A. Torres-Díaz y M. Gual-Díaz. 2014. Licopodios y helechos en el bosque mesófilo de montaña de México. In: Gual-Díaz, M. y A. Rendón-Correa (comps.). Bosques mesófilos de montaña de México: diversidad, ecología y manejo. Comisión Nacional para el Conocimiento y Uso de la Biodiversidad. México, D.F., México. pp. 197-220.

The International Plant Names Index. 2014. Disponible en: http://www.ipni.org. Consultado en julio 2014. 
The Plant List. 2013. Version 1.1. Disponible en: http://www.theplantlist.org/. Consultado en julio 2014.

Thiers, B. (continuamente actualizado). Index Herbariorum: A global directory of public herbaria and associated staff. New York Botanical Garden's Virtual Herbarium. Disponible en: http://sweetgum.nybg.org/ih/.

Tropicos.org. Missouri Botanical Garden. Disponible en: http://www.tropicos.org. Consultado en noviembre 2014.

Valdivia, P. E. 1977. Estudio botánico y ecológico de la región del río Uxpanapa, Veracruz. No. 4. Las epífitas. Biotica 2(1): 55-81.

Vázquez-Torres, M., J. Campos Jiménez y A. Cruz-Pérez. 2006. Los helechos y plantas afines del bosque mesófilo de montaña de Banderilla, Veracruz, México. Polibotánica 22: $63-77$.

Viccon-Esquivel, J. 2009. Riqueza y composición florística de las epífitas vasculares del bosque mesófilo de montaña de las localidades de Atzalan y Zongolica, Veracruz, Tesis de licenciatura. Facultad de Biología, Universidad Veracruzana. Xalapa, México. $72 \mathrm{pp}$.

Wendt, T. 1989. Las selvas de Uxpanapa, Veracruz-Oaxaca, México: evidencia de refugios florísticos cenozoicos. Anales Inst. Biol. Univ. Nac. Méx., Ser. Bot. 58: 29-54.

Williams-Linera, G. 2002. Tree species richness complementarity, disturbance and fragmentation in a Mexican tropical montane cloud forest. Biodivers. \& Conserv. 11: 1825-1843.

Wolf, J. H. D. y A. Flamenco-Sandoval. 2003. Patterns in species richness and distribution of vascular epiphytes in Chiapas, Mexico. J. Biogeogr. 30: 1689-1707.

Wolf, J. H. D. y A. Flamenco-Sandoval. 2005. Distribución y riqueza de epífitas de Chiapas. In: González-Espinosa, M., N. Ramírez-Marcial y L. Ruiz Montoya (coords.). Diversidad biológica en Chiapas. Consejo de Ciencia y Tecnología del Estado de Chiapas, El Colegio de la Frontera Sur, Plaza y Valdés. México, D.F., México. pp. 127-162.

Zamora-Crescencio, P. y G. Castillo-Campos. 1997. Vegetación y flora del municipio de Tlalnelhuayocan, Veracruz. Textos Universitarios, Universidad Veracruzana. Xalapa, México. 82 pp.

Zotz, G. 2013. The systematic distribution of vascular epiphytes-a critical update. Bot. J. Linn. Soc. 171: 453-481. 


\section{APÉNDICE 1}

Helechos y licofitos epífitos de Veracruz.

\section{LYCOPODIACEAE}

\section{LYCOPODIOPHYTA}

Huperzia cuernavacensis (Underw. \& F. E. Lloyd) Holub (=Phlegmariurus cuernavacensis (Underw. \& F. Lloyd) B. Øllg.)

Las Minas: C. Durán E. y M. Bielma 925 (XAL). Ixhuacán de los Reyes: M. Nee 22473 (MEXU). Soteapan: F. Ramírez R. y F. Vázquez B. 1354 (XAL).

Huperzia dichotoma (Jacq.) Trevis.

(=Phlegmariurus dichotomus (Jacq.) W. H. Wagner)

Catemaco: F. Ramírez R. 1381 (XAL). Chiconquiaco: M. Palacios R. 3277 (XAL). Chocamán: O. Sánchez E. 1 (CORU). Jesús Carranza: M. Vázquez T. et al. 700 (UAMIZ). Hidalgotitlán: P.E. Valdivia Q. 149 (MO). San Andrés Tuxtla: J. H. Beaman 5931 (UAMIZ); T. Krömer et al. 1913 (MEXU); D. H. Lorence 3481 (MEXU); S. Sinaca C. 802 (FCME); S. Sinaca C. y F. Chigo S. 650 (MEXU). Zacualpan: C. A. Purpus 1990 (MO).

Huperzia linifolia (L.) Trevis.

(=Phlegmariurus linifolius (L.) B. Øllg.)

Atzalan: T. Krömer 3407 (XALU); T. Krömer y J. Viccon E. 3545 (XAL, XALU). Hidalgotitlán: B. Dorantes 2569 (MO); P. E. Valdivia Q. 1939 (MO); B. Vázquez 217 (MO). Pajapan: J. I. Calzada 11261 (XAL, XALU); T. Krömer y E. Otto 2959 (MEXU). Teocelo: F. Vázquez B. 345 (XAL). Xico: A. Mendoza R. et al. 1848 (UAMIZ). Tlalnelhuayocan: C. Gallardo y L. Lozada 3263 (XAL, XALU); A. Flores P. y J. García F. 889 (XAL).Tlaltetela: C. García M. 1 (CORU). Zacualpan: C. A. Purpus 1991 (MO). Zongolica: I. García G. (CORU). Huperzia myrsinites (Lam.) Trevis. (=Phlegmariurus myrsinites (Lam.) B. Øllg.)

Acajete: J. L. Salazar R. 82 (XALU); L. Sangabriel R. 31, 81 (XALU). Chocamán: Y. Gheno H. 102 (CORU). Emiliano Zapata: P. Hietz y U. Seifert 726 (XAL). Huatusco: Y. Gheno H. s.n. (CORU). Ixhuacán de los Reyes P. Hietz y U. Seifert 795 (XAL). Tlalnelhuayocan: A. Flores P. y J. García F. 900 (XAL, XALU). Xalapa: R. R. Ortega 1355 (XAL).

Huperzia orizabae (Underw. \& F. F. Lloyd) Holub

San Andrés Tuxtla: J. I. Calzada 1548 (MEXU). Soteapan: R. Lira 20 (UAMIZ).

Huperzia pithyoides (Schltdl. \& Cham.) Holub

(=Phlegmariurus pithyoides (Schlecht. \& Cham.) B. Øllg.)

Atoyac: C. Hanaco Rosas s. n. (CORU). Atzalan: T. Krömer 3418 (MEXU). Ixhuatlán del café: M. Velázquez G. 3 (CORU). Misantla: A. Gómez-Pompa 788 (MEXU). San Andrés Tuxtla: J. I. Calzada 67 (UAMIZ); T. Krömer y A. Acebey 2248, 2435 (MEXU); D. H. Lorence 3481 (XAL); A. Rincón et al. 2509 (MEXU, XAL). Santiago Tuxtla: J. H. Beaman y C. Álvarez del Castillo 6280 (MEXU, UAMIZ); T. Krömer y C. A. del Castillo 6280 (MEXU). Soteapan: F. Ramírez R. y F. Vázquez B. 1350 (XAL). Xalapa: C. J. W. Schiede y F. Deppe s.n. (B). Zongolica: T. Krömer 3248 (CORU, MEXU).

Huperzia pringlei (Underw. \& F. E. Lloyd) Holub 
(=Phlegmariurus pringlei (Underw. \& F. E. Lloyd) B. Øllg.)

Acajete: J. L. Salazar R. 104 (XALU); L. Sangabriel R. 33, 92 (XALU). Huatusco: S. Avendaño R. y G. Castillo C. 488 (UAMIZ). San Andrés Tuxtla: J. H. Beaman 5676 (UAMIZ); T. Krömer y A. Acebey 2518, 2259 (MEXU); A. Flores P. s. n. (XAL). Soteapan: J. H. Beaman 6227 (MEXU); R. Lira 40 (UAMIZ).

Huperzia taxifolia (Sw.) Trevis.

(=Phlegmariurus taxifolius (Sw.) Á. Löve \& D. Löve)

Acajete: J. L. Salazar R. 90 (XALU); L. Sangabriel R. 82 (XALU). Atzalan: T. Krömer 3419 (MEXU); A. Mendoza R. et al. 1843 (UAMIZ). Catemaco: R. Lira 178 (MEXU, UAMIZ). Cerro Azul: S. Sinaca C. y F. Chigo S. 591 (ENCB, MEXU). Coatepec: P. Hietz y U. Seifert 818 (XAL). Hidalgotitlán: M. Vázquez T. et al. 586 (UAMIZ). Huatusco: A. Mendoza R. et al. 1211, 2006 (UAMIZ); E. Estrada M. 987 (XAL); S. Levy T. 24 (XAL). Ixhuacán de los Reyes: M. Nee 22473 (MO); P. Hietz y U. Seifert 803 (XAL). Ixtaczoquitlán: L. Benitez D. 17 (CORU). Mecayapan: T. Krömer y E. Otto 2830 (MEXU); F. Ramírez R. y R. Riba 866 (XAL). Orizaba: A. Bozziere G. 12 (CORU); J. F. Caballero E. 3 (CORU). Fortín: A. Valdez L. s. n. (CORU). San Andrés Tuxtla: J. H. Beaman 5586 (MEXU, UAMIZ); J. H. Beaman 5766, 5931 (UAMIZ); J. I. Calzada 126, 11851 (MEXU), 11839 (XAL); R. Cedillo T. 3210 (MEXU, MO); S. Sinaca C. 882 (ENCB, MEXU); T. Krömer et al. 2338 (MEXU, XAL); T. Krömer et al. 4106 (UAMIZ); T. Krömer y A. Acebey 2002 (XAL). Santiago Tuxtla: J. H. Beaman 6410 (MEXU). Soteapan: R. Acosta P. y L. Ceja 1676 (CORU); J. H. Beaman 6232 (MEXU, UAMIZ); J. I. Calzada 12761 (XAL); R. Lira 7 (UAMIZ); R. Ortega et al. 1084 (UAMIZ); R. Riba et al. 1099 (MEXU, UAMIZ); R. Riba et al. 1158 (MEXU, UAMIZ); R. Riba et al. 1183 (UAMIZ); M. Vázquez et al. 3516 (CORU). Tlalnelhuayocan: A. Flores P. s. n. (XAL); A. Flores P. 1014 (XAL); C. Gallardo y L. Lozada 3248 (XAL). Tomatlán: R. Navarro R. 5 (CORU). Xalapa: C. J. W. Schiede y F. Deppe 831 (B). Xico: A. Mendoza R. et al. 1846, 1847 (UAMIZ). P. Hietz y U. Seifert 809 (XAL). Zongolica: H. Flores M. 57 (CORU); I. García G. 4 (CORU); T. Krömer 3038 (CORU, MEXU); J. L. Ramírez C. s.n. (CORU); Y. A. Romero S. 1 (CORU); L. Sánchez T. s.n. (CORU); G. Zamora y M. Hernández H. s. n. (CORU, XAL).

SELAGINELLACEAE

Selaginella extensa Underw.

Tonayan: R. Ortega O. 36 (XAL). Zontecomatlán: M. Nee y K. Taylor 26819 (XAL).

ASPLENIACEAE

\section{POLYPODIOPHYTA}

\section{Asplenium auriculatum Sw.}

Acajete: J. L. Salazar R. 51 (XALU). Atzalan: F. Vázquez B. 2140 (XAL); F. Ventura 11112 (ENCB, XAL); F. Ventura 14373 (MO). Catemaco: R. Lira 184 (MEXU, UAMIZ). Huatusco: F. Ventura 7970 (XALU); F. Ventura 19433 (ENCB). Huayacocotla: L. Ballesteros y F. Ballesteros 404 (UAMIZ, XAL). Mecayapan: F. Ramírez R. 844 (XAL). Misantla: L. Bohs et al. 1700 (MEXU, XAL); A. P. Vovides y R. Riba 293 (XAL). Naolinco: C. A. Purpus s.n. (MO). San Andrés Tuxtla: J. I. Calzada 10638 (XAL); R. Cedillo T. et al. 2975 (MEXU); T. B. Croat y P. Díaz Jiménez 100353 (MO); G. Diggs et al. 2661 (XAL); T. Krömer et al. 1979 (XAL); G. Ibarra M. y S. Sinaca C. 2286 (UAMIZ); A. Rincón et al. 2373 (MEXU). 
Soteapan: R. Lira 207 (ENCB, MEXU, UAMIZ); R. Lira 228 (MEXU, UAMIZ); M. Palacios R. 22, 29 (UAMIZ); F. Ramírez R. 961 (XAL). Teocelo: H. Narave F. 383 (MEXU); C. G. Pringle 7888 (MO); L. Tapia y F. Vázquez B. 33 (XAL). Tlalnelhuayocan: A. Flores P. y J. G. García F. 901 (XAL). Totutla: F. Ventura A. 11399 (XAL). Xico: J. I. Calzada 8327 (XAL). Yecuatla: R. Riba 626 (UAMIZ); S. G. Smith y D. Smith 6025 (XAL); A. P. Vovides y R. Riba 293 (XAL). Zongolica: T. Krömer et al. 3105 (MEXU, UAMIZ).

\section{Asplenium auritum Sw.}

Hidalgotitlán: P. E. Valdivia Q. 187, 235, 611, 1332, 1415, 1820 (XAL). Jesús Carranza: M. Vázquez T. et al. 2400 (MEXU). Las Choapas: A. Franco M. et al. 115 (XAL); E. López P. 309 (XAL). Minatitlán: T. Wendt 2690 (MO). San Andrés Tuxtla: G. Ibarra M. y S. Sinaca C. 2286 (FCME); L. I. Nevling Jr. y A. Gómez-Pompa 2507 (MEXU). Soteapan: R. Riba et al. 1144 (MEXU, UAMIZ).

\section{Asplenium cuspidatum Lam.}

Acajete: J. L. Salazar R. 49 (XALU). Acatlán: F. Ventura 10751 (ENCB); P. Hietz 238 (XAL). Catemaco: R. Lira 132 (MEXU, UAMIZ, XAL); R. Lira 183 (UAMIZ). Huatusco: S. Avendaño E. et al. 361 (IEB, XAL). Huayacocotla: L. Ballesteros y F. Ballesteros 369 (UAMIZ); L. Ballesteros y F. Ballesteros 405 (UAMIZ, XAL). Mecayapan: T. Krömer y E. Otto 2870 (MEXU). Miahuatlán: F. Ventura 2946 (ENCB). Pajapan: M. Nee y J. I. Calzada 22764 (XAL). San Andrés Tuxtla: T. Krömer et al. 1915 (MEXU, XAL), T. Krömer et al. 2326 (MEXU); T. Krömer et al. 2708 (MEXU); G. Ibarra M. y S. Sinaca C. 2286 (XAL); S. Sinaca C. y F. Chigo S. 601 (ENCB). Tlalnelhuayocan: T. Krömer 3933, 3950 (MEXU). Yecuatla: C. I. Carvajal H. 218 (MEXU). Zongolica: T. Krömer et al. 3054 (MEXU).

Asplenium feei Kunze ex Fée

Soteapan: R. Lira 16 (ENCB, MEXU, UAMIZ, XAL).

Asplenium formosum Willd.

Catemaco: F. Ponce C. y C. Álvarez del Castillo 346 (MO). Hidalgotitlán: P. E. Valdivia Q. 224 (UAMIZ, XAL); M. Vázquez V. et al. 921 (UAMIZ). Tlacotepec de Mejía: C. A. Purpus 2178 (MO).

Asplenium fragrans Sw.

Acatlán: F. Ventura A. 10751 (XAL). Coatepec: M. Cházaro y L. E. Castillo Ch. 6794 (MO). San Andrés Tuxtla: T. B. Croat y P. Díaz J. 100343 (MO); T. Krömer y A. Acebey 2434 (MEXU). Zongolica: T. Krömer et al. 3112 (MEXU, UAMIZ).

\section{Asplenium harpeodes Kunze}

Atzalan: T. Krömer 3473 (MEXU, UAMIZ). Huatusco: F. Ventura 7962 (ENCB, MEXU). Jalacingo: F. Ventura 343 (ENCB). Jilotepec: F. Ventura A. 17061 (ENCB, IEB, MEXU); F. Ventura 20293 (ENCB, IEB, MEXU). Tlalnelhuayocan: T. Krömer 3935 (XALU). Xico: H. Narave F. 383 (XAL).

Asplenium laetum Sw.

Huatusco: F. Ventura A. 19433 (XAL).

Asplenium miradorense Liebm.

Acajete: J. L. Salazar R. 67 (XALU). Atzalan: F. Ventura A. 11613 (IEB). Huatusco: R. Chicatto G. 4 (CORU). San Andrés Tuxtla: J. H. Beaman 5812, 5942 (UAMIZ). Soteapan: R. Ortega et al. 1142 (ENCB, MEXU, UAMIZ). Totutla: F. Ventura 5118 (ENCB, XALU). Zongolica: T. Krömer 3034 (XALU). 
Asplenium monanthes L.

Calcahualco: A. Rincón G. y C. Durán E. 2697 (MEXU). Jalacingo: F. Ventura 43 (ENCB). Zongolica: T. Krömer et al. 3031, 3106 (MEXU).

Asplenium monodon Liebm.

Soteapan: R. Riba, B. Pérez G. y R. Lira 1144 (ENCB).

Asplenium praemorsum $\mathrm{Sw}$.

Acatlán: F. Ventura 4916 (ENCB). Huatusco: S. Avendaño R. et al. 361 (MEXU, MO); E. R. de la Sota 4003 (ENCB); A. Mendoza R. et al. 1217 (UAMIZ). Ixhuatlancillo: J. S. Miller y R. Torres C. 2979 (MEXU). Ixtaczoquitlán: E. M. Navarro 18 (CORU). Naolinco: F. Ventura 17002 (ENCB, MEXU). Orizaba: D. M Palacios S. 4 (CORU). Pajapan: M. Nee y J. I. Calzada 22764 (MEXU). San Andrés Tuxtla: G. Diggs et al. 2694 (XAL); G. Ibarra M. y S. Sinaca C. 2286 (MO); G. Ibarra M. et al. 3015 (UAMIZ); G. Ibarra M. et al.3016 (MEXU, UAMIZ); M. Sousa S. 3448 (MEXU); S. Sinaca C. 920 (MO, UAMIZ); S. Sinaca C. y F. Chigo S. 601 (MEXU, UAMIZ). Teocelo: F. Ventura 16808 (ENCB, IEB, MEXU). Vega de Alatorre: B. Guerrero C. y J. I. Calzada 1800 (XAL). Zongolica: F. Vázquez B. 1386 (XAL). Asplenium pteropus Kaulf.

Atzalan: T. Krömer y J. Viccon E. 3503 (MEXU); E. Ventura A. 11613 (MEXU). San Andrés Tuxtla: T. Krömer y A. Acebey 2503 (MEXU); T. Krömer et al. 2583 (MEXU).

Asplenium rutaceum Willd.

Soteapan: R. Lira 217 (ENCB, UAMIZ, XAL).

Asplenium salicifolium L.

Hidalgotitlán: P. E. Valdivia Q. 1851 (XAL). Jesús Carranza: A. Mendoza R. et al. 1178 (IEB, UAMIZ).

Asplenium serra Langsd. \& Fisch.

Acajete: J. L. Salazar R. 52 (XALU). Chocamán: P. E. Valdivia Q. 2197 (XAL). Coatepec: P. Hietz y V. Seifert 848 (MEXU). Huatusco: F. Ventura A. 8265 (ASU, ENCB, IEB, MEXU, UAMIZ, XAL). Huayacocotla: L. Ballesteros y F. Ballesteros 449 (XAL). Minatitlán: T. Wendt et al. 3710 (ENCB). Tlalnelhuayocan: F. Ventura 12350 (ENCB, MEXU). Zongolica: T. Krömer et al. 3277 (MEXU); A. Rincón G y C. Durán E. 1329 (MEXU).

\section{Asplenium serratum L.}

Catemaco: P. Hietz y U. Seifert 892 (XAL); R. Riba et al. 1217 (ENCB, UAMIZ). Hidalgotitlán: Brigada Dorantes 2559 (MEXU, MO); P. E. Valdivia Q. 329 (XAL); P. E. Valdivia Q. 1414 (IEB, UAMIZ); R. Riba y B. Pérez G. 809 (MO, XAL). Las Choapas: A. Mendoza R. et al. 917 (IEB, UAMIZ). Minatitlán: T. Wendt y M. Ishiki I. 5871 (MEXU). San Andrés Tuxtla: A. Mendoza R. et al. 849 (UAMIZ); R. Riba 1937 (UAMIZ).

Asplenium sessilifolium Desv.

Huayacocotla: L. Ballesteros y F. Ballesteros 382 (UAMIZ, XAL, XALU). Tepetzintla: G. Castillo C. et al. 2494 (MEXU, XAL).

Asplenium sphaerosporum A. R. Sm.

Banderilla: P. Hietz 179 (XAL). Huatusco: A. Mendoza R. et al. 1206 (IEB, UAMIZ); F. Ventura 7970 (ENCB, MEXU); F. Vázquez B. 1365 (XAL). Ixhuatlancillo: J. Miranda B. 19 (CORU). Jilotepec: D. S. Conant 705 (MEXU). Naolinco: F. Ventura 15058 (ENCB, IEB, MEXU). Orizaba: C. G. Pringle 5584 (MO). Xalapa: J. R. Galindo G. 6 (XAL). Zongolica: M. Martínez s.n. (FCME). 


\section{BLECHNACEAE}

Blechnum ensiforme (Liebm.) C. Chr.

Catemaco: R. Lira 114 (UAMIZ). Pajapan: T. Krömer y E. Otto 2966 (MEXU). Soteapan: R. Riba et al. 1173 (ENCB, MEXU, UAMIZ); M. Palacios R. 19 (UAMIZ).

Blechnum fragile (Liebm.) C. V. Morton \& Lellinger

Chocamán: M. Nee 23334 (XAL).

\section{DRYOPTERIDACEAE}

Bolbitis bernoullii (Kuhn ex Christ) Ching (hemiepífita)

(=Mickelia bernoullii (Kuhn ex Christ) R. C. Moran, Labiak \& Sundue)

Amatitlán: R. Riba 1696 (ENCB, UAMIZ, XAL). Atzalan: T. Krömer y J. Viccon E. 3449 (MEXU). Catemaco: J. H. Beaman 5926 (UAMIZ); R. Lira 165, 194 (MEXU, UAMIZ); D. H. Lorence y T. P. Ramamoorthy 3292 (MEXU); A. Mendoza R. et al. 617, 618 (UAMIZ); R. Riba 1255 (UAMIZ). Hidalgotitlán: J. Dorantes et al. 4136 (UAMIZ); Brigada Dorantes 2460, 2773, 2907 (MEXU, XAL). Las Choapas: A. Mendoza R. et al. 899, 914 (UAMIZ). San Andrés Tuxtla: R. Cedillo T. 2556 (MEXU); T. Krömer et al. 1917 (MEXU); G. Ibarra M. et al. 345 (MEXU); G. Ibarra M. y S. Sinaca C. 2338 (MEXU); G. Ibarra M. y N. Pérez N. 3097 (MEXU); A. Mendoza R. et al. 843 (UAMIZ); A. Pérez P. 40 (MEXU); T. P. Ramamoorthy y G. Ibarra M. 3266 (MEXU); R. Riba 1963 (UAMIZ).

Bolbitis serratifolia (Mert. ex Kaulf.) Schott (hemiepífita)

Las Choapas: A. Mendoza R. et al. 913, 921 (UAMIZ). San Andrés Tuxtla: R. Riba 419 (ENCB, UAMIZ).

Dryopteris patula (Sw.) Underw.

Acatlán: F. Ventura A. 10749 (ASU, MEXU). Huatusco: A. Mendoza R. et al. 1205 (IEB, UAMIZ). Ixhuatlancillo: S. M. Chambé L. 2 (CORU). Tlalnelhuayocan: A. Ventura A. 15767 (IEB, MEXU).

Elaphoglossum affine (M. Martens \& Galeotti) T. Moore

Calcahualco: F. Vázquez B. 1673 (XAL).

Elaphoglossum erinaceum (Fée) T. Moore

Catemaco: R. Lira 121 (UAMIZ). San Andrés Tuxtla: J. I. Calzada 572 (MEXU). Soteapan: R. Lira 9, 223 (UAMIZ); M. Palacios R. 18 (UAMIZ); R. Riba 1101 (IEB, MEXU, UAMIZ). Elaphoglossum glabellum J. Sm.

Mecayapan: T. Krömer y E. Otto 2871 (MEXU).

Elaphoglossum glaucum T. Moore

Juchique de Ferrer: G. Castillo C. et al. 1877 (XAL). Landero y Coss: P. E. Valdivia Q. 2212 (IEB, MEXU); P. E. Valdivia Q. 92507 (XAL).

Elaphoglossum guatemalense (Klotzch) T. Moore

Atzalan: T. Krömer \& J. Viccon E. 3474 (UAMIZ); Catemaco: A. Gómez-Pompa et al. 5364 (UAMIZ). Mecayapan: T. Krömer y E. Otto 2827, 2829, 2977 (MEXU). San Andrés Tuxtla: G. Ibarra M. et al. 3012 (MEXU, MO, UAMIZ); T. Krömer y A. Acebey 1988, 2425, 2506, 2519, 2533 (MEXU). Soteapan: R. Lira 11 (UAMIZ).

Elaphoglossum leebrowniae Mickel

Atzalan: T. Krömer et al. 3310 (MEXU). 
Elaphoglossum lonchophyllum (Fée) T. Moore

Atzalan: T. Krömer y J. Viccon E. 3475 (MEXU). Coscomatepec: F. Ventura A. 8726 (ENCB, IEB, UAMIZ). Orizaba: E. B. Copeland 158 (MEXU). Tlalnelhuayocan: T. Krömer 3951 (XALU).

Elaphoglossum muscosum (Sw.) T. Moore

San Andrés Tuxtla: T. Krömer y A. Acebey 2430 (MEXU).

Elaphoglossum obscurum (E. Fourn.) C. Chr.

Jilotepec: R. Riba 953 (ENCB). Misantla: W. J. Kress et al. 749 (MEXU).

Elaphoglossum paleaceum (Hook \& Grev.) Sledge

Coscomatepec: F. Ventura 8724 (MEXU).

Elaphoglossum peltatum (Sw.) Urb.

Acajete: J. L. Salazar R. 109 (XALU). Atzalan: T. Krömer y A. Viccon E. 3480 (MEXU). Catemaco: A. Gómez Pompa et al. 5441 (XAL); R. Lira 111, 189 (UAMIZ). Chiconquiaco: A. Gómez-Pompa y R. Riba 392 (MEXU). Huatusco: J. I. Calzada 7981 (ENCB, MEXU, XAL). Huayacocotla: L. Ballesteros y H. Morales 500 (XAL). Jalacingo: F. Ventura 340, 9212 (ENCB). Mecayapan: T. Krömer y E. Otto 2838 (MEXU). San Andrés Tuxtla: J. I. Calzada 547 (MEXU); T. Krömer y A. Acebey 1986 (MEXU); T. Krömer y A. Pérez P. 2323 (MEXU); A. Rincón G. et al. 2449 (MEXU); S. Sinaca C. et al. 960 (MEXU, UAMIZ). Soteapan: R. Lira 26 (ENCB, UAMIZ); M. Nee et al. 24657 (XAL); R. Ortega et al. 1111 (UAMIZ, XAL); R. Riba et al. 1085, 1157 (UAMIZ). Teocelo: F. Ventura 7331 (ENCB). Xico: J. I. Calzada 8341 (MEXU); M. Cházaro B. y P. Cházaro H. 4032 (IEB, XAL); P. Tenorio L. 15543 (MEXU). Zongolica: T. Krömer et al. 3113 (MEXU).

Elaphoglossum petiolatum (Sw.) Urb.

Las Vigas de Ramírez: S. Caughlan T. et al. 337 (MEXU); G. Diggs at al. 2120 (MEXU). Pajapan: T. Krömer y E. Otto 2960 (MEXU). San Andrés Tuxtla: T. Krömer y A. Acebey 2087, 2498 (MEXU); T. Krömer et al. 2374, 2392 (MEXU); T. Krömer y A. Pérez P. 2324 (MEXU); T. Krömer y E. Velasco 2041 (MEXU). Tatahuicapan de Juárez: T. Krömer et al. 3670 (MEXU). Zongolica: T. Krömer et al. 3080 (MEXU).

Elaphoglossum rubescens (Kuhn) Christ.

San Andrés Tuxtla: L. I. Nevling y A. Gómez-Pompa 2523 (MEXU).

Elaphoglossum sartorii (Liebm.) Mickel

Atzalan: T. Krömer y J. Viccon E. 3544 (MEXU). Catemaco: R. Lira 185 (UAMIZ, XAL). Chiconquiaco: A. Gómez-Pompa y R. Riba 433 (UAMIZ). San Andrés Tuxtla: J. H. Beaman y Álvarez 5693 (UAMIZ); T. Krömer y A. Acebey 1989 (MEXU). Soteapan: R. Lira 22 (ENCB, MEXU, UAMIZ, XAL); R. Lira 224 (MEXU, UAMIZ); R. Lira 229 (MEXU, UAMIZ). Xalapa: J. I. Calzada 1944 (XAL). Zongolica: T. Krömer 3229 (citada por VicconEsquivel, 2009).

Elaphoglossum vestitum (Schltdl. \& Cham.) T. Moore

Acajete: L. Sangabriel R. 23 (XALU). Atzalan: T. Krömer et al. 3342 (MEXU). Catemaco: R. Lira 120 (UAMIZ); R. Lira 180 (MEXU, UAMIZ). Coscomatepec: T. B. Croat y D. P. Hannon 63105 (MEXU, MO). Huatusco: S. Avendaño y R. Ortega 262 (MEXU). Mecayapan: T. Krömer y E. Otto 2851 (MEXU). Misantla: D. S. Barrington 391 (MEXU); D. S. Conant 753 (MEXU). San Andrés Tuxtla: T. Krömer y A. Acebey 1987 (MEXU); T. Krömer y E. Velasco S. 1934 (MEXU); T. Krömer et al. 2337 (MEXU); A. Pérez P. et al. 10 
(MEXU); Sousa 3454 (MEXU). Soteapan: R. Lira 23, 227 (MEXU, UAMIZ). Xalapa: C. J. W. Schiede 766 (BGBM). Xico: P. E. Valdivia Q. 2223 (XAL). Zongolica: T. Krömer et al. 3040 (MEXU); P. E. Valdivia Q. 2136 (XAL).

Elaphoglossum viride (E. Fourn) C. Chr.

Misantla: D. S. Conant 752 (MEXU). San Andrés Tuxtla: T. Krömer y A. Acebey 2200, 2206, 2426, 2454 (MEXU).

Polybotrya caudata Kunze (hemiepífita)

Las Choapas: A. Franco et al. 108 (XAL).

Polybotrya polybotryoides (Baker) Christ (hemiepífita)

Mecayapan: G. Castillo C. et al. 12599 (XAL).

\section{HYMENOPHYLLACEAE}

Hymenophyllum asplenioides (Sw.) Sw.

Atzalan: T. Krömer 3467 (XALU). Soteapan: R. Lira 39 (MEXU, UAMIZ).

Hymenophyllum brevistipes Liebm.

Atzalan: T. Krömer 3542 (XALU).

Hymenophyllum crispum Kunth

Acatlán: P. Hietz, 292 (XAL). Misantla: R. M. Fonseca J. s.n. (IEB).

Hymenophyllum fendlerianum J. W. Sturm

Atzalan: T. Krömer 3543 (MEXU).

Hymenophyllum fucoides Sw.

Acajete: L. Sangabriel R. 39 (XALU). Chiconquiaco: F. Ventura A. 7582 (MEXU, NY). San Andrés Tuxtla: T. Krömer y A. Acebey 2197, 2424 (MEXU).

Hymenophyllum lanatum Fée

San Andrés Tuxtla: T. Krömer et al. 2031, 2032 (MEXU, XAL, XALU); T. Krömer y A. Acebey 2481 (MEXU); T. Krömer et al. 2588 (MEXU).

Hymenophyllum maxonii Christ ex C. V. Morton

San Andrés Tuxtla: T. Krömer y A. Acebey 2422 (XAL, XALU). Santiago Tuxtla: J. H. Beaman y C. Álvarez del C. 5773 (MEXU, UAMIZ). Soteapan: J. H. Beaman 5447 (UAMIZ).

Hymenophyllum myriocarpum Hook.

Acajete: L. Sangabriel R. 95-B (XALU). Mecayapan: T. Krömer y E. Otto 2889 (MEXU).

Xico: J. I. Calzada 8340 (XAL).

Hymenophyllum polyanthos (Sw.) Sw.

Acajete: J. L. Salazar R. 66 (XALU). Acatlán: P. Hietz 231 (XAL). Atzalan: T. Krömer y J. Viccon E. 3468 (MEXU). Catemaco: R. Riba y B. Pérez G. 1265 (UAMIZ). Coatepec: M. Cházaro B. y L. E. Castillo C. 6787 (XAL): Huatusco: A. Mendoza R. et al. 1213 (IEB, UAMIZ). Mecayapan: T. Krömer y E. Otto 2891 (MEXU). San Andrés Tuxtla: E. Arguelles 985 (MEXU); T. Krömer y A. Acebey 1985, 2097 (MEXU, XAL), T. Krömer y A. Acebey 2423 (MEXU); T. Krömer et al. 2371 (MEXU); S. Sinaca C. et al. 985 (MEXU, UAMIZ). Soteapan: L. Bohs et al. 1691 (MEXU); M. Nee et al. 25029, 25072 (XAL); F. Ramírez R. 1548 (XAL); R. Riba et al. 1073-80A, 1078-80, 1089-bis (UAMIZ). Tlalnelhuayocan: A. Flores P. 996 (XAL). Totutla: G. Rodríguez S. 4P (UAMIZ). Zacualpan: C. A. Purpus 4368 (MO). 
Hymenophyllum pulchellum Schltdl. \& Cham.

Acajete: J. L. Salazar R. 72 (XALU). San Andrés Tuxtla: T. Krömer et al. 2370, 2587 (MEXU). Zongolica: T. Krömer 3133 (citada por Viccon-Esquivel, 2009).

Hymenophyllum tegularis (Desv.) Proctor \& Lourteig

Xico: P. Hietz 419 (XAL).

Hymenophyllum trapezoidale Liebm.

Atzalan: T. Krömer 3439 (XALU). Mecayapan: F. Ramírez R. y R. Riba 861 (XAL). San Andrés Tuxtla: J. H. Beaman y C. Álvarez del C. 5773 (UAMIZ). Soteapan: R. Lira 29 (MEXU, UAMIZ); M. Palacios R. 34 (MEXU, UAMIZ); M. Palacios R. 35 (UAMIZ); R. Riba, B. Pérez G. y L. de León 1119 (UAMIZ). Xalapa: C. G. Pringle 8157 (MO). Zacualpan: C. A. Purpus 1985 (MO).

Hymenophyllum tunbridgense (L.) Sm.

Acatlán: P. Hiertz 290 (XAL). Chiconquiaco: J. I. Calzada 9341 (XAL). Soteapan: R. Riba et al. 1097-bis (UAMIZ).

Trichomanes bucinatum Mickel \& Beitel

Acatlán: P. Hietz y U. Seifert 458 (XAL). Xalapa: C. G. Pringle 10809 (XAL). Xico: P. Hietz y U. Seifert 724 (XAL).

Trichomanes capillaceum L.

(=Polyphlebium capillaceum (L.) Ebihara \& Dubuisson)

Atzalan: T. Krömer et al. 3312 (MEXU, UAMZ); S. Zamudio R. y P. Zamora 8465 (IEB, MEXU, UAMIZ); S. Zamudio R. y P. Zamora 8470 (IEB). Banderilla: V. Hernández H. 12 (XALU); T. Krömer et al. 3187 (UAMIZ). Catemaco: T. Krömer et al. 2572 (MEXU); R. Lira 169 (ENCB, MEXU; UAMIZ); R. Riba y B. Pérez G. 1264 (UAMIZ). Chiconquiaco: A. Gómez Pompa y R. Riba 426 (MEXU, UAMIZ). Mecayapan: T. Krömer y E. Otto 2997 (XAL:); F. Ramírez R. 761 (XAL). Misantla: D. S. Conant 829 (MEXU). Tlalnelhuayocan: A. Flores P. y J. G. García F. 892 (XAL). Soteapan: M. Palacios R. 33 (UAMIZ). Xalapa: C. G. Pringle 8173 (MEXU, MO).

Trichomanes collariatum Bosch

Catemaco: P. Hietz y U. Seifert 1019 (XAL); M. Nee y G. Schatz 19937 (XAL); R. Riba et al. 1232 (UAMIZ). Hidalgotitlán: Brigada Dorantes 2452, 2535 (MO); J. Dorantes 2726 (UAMIZ, XAL). Las Choapas: A. Franco et al. 111 (XAL); A. Mendoza R. et al. 902 (UAMIZ). San Andrés Tuxtla: T. B. Croat y D. P. Hannon 63130 (XAL, MO); T. Krömer y A. Acebey 2064, 2115 (XAL); A. Pérez P. et al. 9 (MEXU); T. Krömer y A. Acebey 2115, 2740 (MEXU).

Trichomanes crispum L.

Acatlán: P. Hiertz 232 (XALU).

Trichomanes galeottii E. Fourn.

Pajapan: T. Krömer y E. Otto 2962 (MEXU, XAL).

Trichomanes godmanii Hook.

Las Choapas: A. Franco M. et al. 95 (XAL, XALU).

Trichomanes hymenoides Hedw.

Catemaco: R. Lira 199 (UAMIZ). Mecayapan: T. Krömer y E. Otto 2932 (MEXU, XAL).

Soteapan: R. Riba et al. 1156, 1159 (UAMIZ). Zacualpan: C. A. Purpus 2929 (MO).

Trichomanes hymenophylloides Bosch 
Atzalan: T. Krömer et al. 3373 (MEXU). San Andrés Tuxtla: T. Krömer y A. Acebey 2521 (MEXU).

Trichomanes krausii Hook. \& Grev.

Hidalgotitlán: M. Vásquez et al. 789 (UAMIZ, XAL). Misantla: W. J. Kress et al. 824 (MEXU). Soteapan: R. Riba et al. 1155 (UAMIZ). Xalapa: C. G. Pringle 10809 (ASU, ENCB).

\section{Trichomanes membranaceum L.}

Las Choapas: A. Mendoza R. et al. 905 (UAMIZ).

Trichomanes ovale (E. Fourn.) Wess. Boer

San Andrés Tuxtla: T. Krömer y A. Acebey 2736 (MEXU).

Trichomanes polypodioides L.

Atzalan: T. Krömer y J. Viccon E. 3469 (MEXU); F. Ventura A. 17219 (ENCB, IEB, MEXU). Soteapan: R. Lira 10 (ENCB, MEXU, UAMIZ); R. Riba 1076-80B (UAMIZ). Totutla: F. Ventura A. 11077 (ASU, ENCB, MEXU).

Trichomanes pyxidiferum L.

(=Polyphlebium pyxidiferum (L.) Ebihara \& Dubuisson)

Banderilla: T. Krömer et al. 3189 (UAMIZ). Tlalnelhuayocan: F. Ventura A. 18202 (IEB, MEXU, UAMIZ).

Trichomanes radicans Sw.

(=Vandenboschia radicans (Sw.) Copel.)

Acajete: J. L. Salazar R. 56 (XALU). Acatlán: P. Hietz y U. Seifert 468 (XAL). Atzalan: S. Zamudio R. y P. Zamora 8464 (MEXU, XAL). Banderilla: T. Krömer et al. 3180 (MEXU); T. Krömer et al. 3750 (UAMIZ). Catemaco: R. Lira 109 (ENCB, MEXU, UAMIZ), R. Lira 119, 152 (UAMIZ); M. Nee y G. Schatz 19885 (MEXU). Chiconquiaco: A. Gómez Pompa y R. Riba 350, 414 (MEXU); R. Riba 621 (ENCB, UAMIZ); R. Riba 648 (MEXU). Huayacocotla: F. Ramírez R. 630 (XAL). San Andrés Tuxtla: T. Krömer y A. Pérez P. 2750 (MEXU); A. Rincón et al. 2393 (XAL). Soteapan: R. Ortega et al. 1152 (UAMIZ); M. Palacios R. 32 (MEXU, UAMIZ); R. Riba et al. 1098-80 (UAMIZ). Tepetzintla: G. Castillo C. et al. 2541 (XAL).

Trichomanes reptans Sw.

(=Didymoglossum reptans (Sw.) C. Presl)

Atzalan: T. Krömer 3930 (XALU). Banderilla: T. Krömer et al. 318 (UAMIZ). Catemaco: R. Lira 199 (ENCB, MEXU). Tlalnelhuayocan: A. Flores P. y J. García F. 893 (XAL). San Andrés Tuxtla: T. Krömer y A. Acebey 2369, 2552 (MEXU); G. Ibarra M. et al. 2422a (MEXU). Xalapa: C. G. Pringle 10809 (XAL). Zongolica: T. Krömer et al. 3044 (MEXU).

\section{LOMARIOPSIDACEAE}

\section{Lomariopsis mexicana Holttum}

San Andrés Tuxtla: T. Krömer y A. Acebey 2490 (MEXU); S. H. Sohmer 9460 (MEXU).

\section{Lomariopsis recurvata Fée}

Catemaco: R. Riba et al. 1197 (UAMIZ). Hidalgotitlán: Brigada Dorantes 2908 (MEXU, MO); R. Riba y B. Pérez G. 793 (MEXU). Las Choapas: E. López P. 122, 166, 177, 599 (XAL). San Andrés Tuxtla: T. Krömer y A. Pérez P. 2764 (MEXU); R. Riba 420 (MEXU). Soteapan: A. Mendoza R. et al 1189 (UAMIZ). 
Mendoza-Ruiz et al.: Helechos y licofitos epífitos de Veracruz, México

Nephrolepis exaltata (L.) Schott

San Andrés Tuxtla: A. Mendoza R. et al. 852 (UAMIZ).

Nephrolepis pendula (Raddi) J. Sm.

Hidalgotitlán: Brigada Dorantes 2768 (MEXU, UAMIZ); P. E. Valdivia Q. 428 (XAL). Minatitlán: T. L. Wendt y W. H. Hernández G. 5488 (NY).

Nephrolepis undulata (Afzel. ex Sw.) J. Sm.

Orizaba: A. Mendoza R. et al. 1164 (UAMIZ).

OLEANDRACEAE

Oleandra articulata (Sw.) Presl

Hidalgotitlán: T. Wendt et al. 3846 (MEXU).

\section{POLYPODIACEAE}

Campyloneurum amphostenon (Kunze ex Klotzsch) Fée

Acajete: L. Sangabriel R. 89 (XALU). Acatlán: F. Ventura A. 11848 (ENCB, IEB). Altotonga: F. Ventura A. 18534 (IEB). Calcahualco: J. L. Martínez y F. Vázquez B. 283 (IEB).

Campyloneurum angustifolium (Sw.) Fée

Acajete: J. L. Salazar R. 59 (XALU); L. Sangabriel R. 50, 110 (XALU). Acatlán: F. Ventura A. 7295 (ENCB); F. Ventura A. 19134 (ENCB, MEXU, MO). Altotonga: F. Ventura A. 110 (ENCB, MO); F. Ventura A. 18534 (MEXU). Amatlán de los Reyes: J. Rzedowski 18874 (ENCB). Atzalan: F. Ventura A. 84 (ENCB). Calcahualco: J. L. Martínez P. y F. Vázquez B. 283 (MEXU). Catemaco: J. H. Beaman 6476 (UAMIZ); R. Cedillo T. 3402 (MEXU); R. Hernández 1386 (MEXU); R. Lira 145 (ENCB, MEXU, UAMIZ); R. Lira 170 (ENCB, UAMIZ); A. Mendoza R. et al. 617b (UAMIZ); R. Riba et al. 705 (MEXU, UAMIZ); R. Riba et al. 1223 (MEXU, UAMIZ); A. Torres S. y A. Campos V. 18 (MEXU); J. Rzedowski 20356 (ENCB, MEXU); S. H. Sohmer 9465 (MEXU); J. van Rooden 810 (MEXU); Chiconquiaco: J. Calzada 7903 (ENCB); R. Riba 628 (UAMIZ); F. Ventura A. 5125, 7921 (ENCB, XALU). Coetzala: G. Diggs, M. Nee y G. Schatz 2720 (ENCB). Comapa: A. Mendoza R. et al. 1204 (UAMIZ). Córdoba: T. B. Croat 39608 (MO). Cuitláhuac: R. Riba 415 (MEXU). Fortín: T. B. Croat 39427 (MEXU); T. B. Croat 39459 (MEXU, MO); J. T. Mickel 736 (ENCB); R. Riba 1561 (UAMIZ). Hidalgotitlán: P. E. Valdivia Q. 324 (IEB, UAMIZ), P. E. Valdivia Q. 426 (UAMIZ, XAL); P. E. Valdivia Q. 1380 (UAMIZ); T. Wendt et al. 2739 (MEXU). Huatusco: S. Avendaño R. 420 (IEB); S. Avendaño R. y J. I. Calzada 429 (XAL); E. R. de la Sota 4039 (ENCB); A. Mendoza R. et al. 1212 (UAMIZ); J. Rzedowski 18970, 18986 (ENCB). Huayacocotla: L. Ballesteros y F. Ballesteros 407 (UAMIZ); A. Mendoza R. et al. 1667 (UAMIZ). Huiloapan de Cuauhtémoc: J. I. Calzada 8573 (XAL). Jalcomulco: G. Castillo C. y W. Bussey 3013 (XAL). Jesús Carranza: A. Mendoza R. et al. 1177 (UAMIZ); M. Vásquez et al. 708 (UAMIZ); T. Wendt y A. Villalobos C. 4454 (MEXU, MO). Orizaba: A. Mendoza R. et al. 1165 (UAMIZ); Stone 3062 (MO). Pajapan: M. Nee y J. I. Calzada 22768 (MEXU). Río Blanco: P. Rossiles B. y M. V. Dávila J. 20 (CORU). San Andrés Tuxtla: J. I. Calzada 1547 (ENCB, MO, UAMIZ); J. J. den Held y F. A. van Rhijn s.n. (MEXU); G. Ibarra M. 307 (MEXU); H. Kennedy y C. Horvitz s.n. (MEXU); D. H. Lorence 3477 (MO, XAL); T. P. Ramamoorthy y G. Ibarra M. 3268 (MEXU); R. Riba 836 (MO); S. Sinaca C. 921 (MEXU, MO, UAMIZ); S. Sinaca C. y P. Ventura F. 1490 (MEXU, MO, UAMIZ). 
Soteapan: A. Mendoza R. et al. 1185, 1190, 1193 (UAMIZ); R. Riba et al. 1135 (MEXU, UAMIZ). Tehuipango: A. Rincón G. y C. Durán E. 1293 (MEXU). Tomatlán: E. Ventura A. 3825 (ENCB, MEXU). Uxpanapa: R. Riba 853 (UAMIZ). Xalapa: G. Castillo C. et al. 44 (ENCB), E. Ronzon 6 (IEB). Xico: H. Narave F. 321 (MEXU). Yecuatla: R. Ortega 2017 (ENCB). Zongolica: R. Riba 1308 (ENCB).

Campyloneurum phyllitidis (L.) C. Presl

Agua Dulce: A. Lot H. 2212 (MEXU). Catemaco: R. Riba et al. 712 (MEXU, UAMIZ). Coscomatepec: E. R. de la Sota 4047 (MEXU). Amatlán de los Reyes: J. Rzedowski 18888 (MEXU). Fortín: T. B. Croat 39430 (MO). Misantla: A. Gómez-Pompa y R. Riba 255 (MEXU). Texistepec: J. Chavelas P. et al. Es-2710 (MEXU). Soteapan: R. Lira y B. PérezGarcía 1142 (MEXU). Tlapacoyan: F. Ventura A. 18939 (ENCB, MEXU, UAMIZ).

Campyloneurum repens (Aubl.) C. Presl

San Andrés Tuxtla: J. H. Beaman 5734 (MEXU).

Campyloneurum serpentinum (Christ) Ching

Catemaco: R. Lira 128, 173 (UAMIZ). Hidalgotitlán: R. Riba y B. Pérez G. 807 (XAL). Las Choapas: A. Mendoza R. et al. 911 (UAMIZ).

\section{Campyloneurum xalapense Fée}

Alto Lucero de Gutiérrez Barrios: G. Castillo C. y F. Vázquez B. 1354 (XAL). Catemaco: L. Cortés A. s.n. (MEXU); A. Gómez-Pompa et al. 5371 (UAMIZ); R. Lira 128 (MEXU); R. Lira 130 (UAMIZ); R. Lira 143 (UAMIZ); R. Lira 164 (MEXU, UAMIZ); R. Lira 177 (UAMIZ); R. Lira 193 (MEXU, UAMIZ). Chocamán: F. Ventura 16285 (ASU, ENCB, IEB, MEXU). Coscomatepec: T. B. Croat y D. P. Hannon 63103 (MEXU, MO); E. R. de la Sota 4047 (ENCB). Fortín: T. B. Croat 39386, 39413 (MO); R. Riba 1560 (UAMIZ). Huatusco: E. R. de la Sota 4004 (ENCB); F. Ventura 4797 (ENCB, MEXU, XALU). Ixhuatlán del Café: F. Ventura 16262 (ENCB, IEB, MEXU); F. Ventura A. 16263 (XAL). Juchique de Ferrer: G. Castillo C. et al. 1733 (XAL). Naolinco: J. I. Calzada 9397 (ENCB, MEXU). Orizaba: T. B. Croat 44046 (MO). Pajapan: J. Calzada 426 (MO). San Andrés Tuxtla: J. H. Beaman 5762 (MEXU, UAMIZ); S. Sinaca C. y F. Chigo S. 711 (UAMIZ); S. Sinaca C. y G. Ibarra M. 1692 (MEXU, UAMIZ). Soteapan: R. Riba et al. 1161 (UAMIZ). Tlacotepec de Mejía: C. A. Purpus 2163 (MO). Totutla: F. Ventura A. 10449 (IEB, MEXU, XAL); F. Ventura 11402 (ENCB, IEB); F. Ventura 16005 (ENCB). Xalapa: A. Rebolledo V. s. n. (ENCB). Xico: L. Tapia y F. Vázquez B. 18 (XAL); L. Zelaya et al. 70 (ENCB, IEB, UAMIZ); A. Zlotnik 43 (UAMIZ). Yecuatla: R. Riba 544 (MEXU, UAMIZ); R. Riba 630 (UAMIZ).

Cochlidium linearifolium (Desv.) Maxon ex C. Chr.

Tatahuicapan de Juárez: T. Krömer et al. 3664 (UAMIZ). Xico: M. Cházaro B. y P. Hernández de Cházaro 4035 (XAL); P. Tenorio L. 15498, 15545 (MEXU).

Cochlidium rostratum (Hook.) Maxon ex C. Chr.

San Andrés Tuxtla: T. Krömer y A. Acebey 2077, 2432, 2552 (XAL). Soteapan: R. Lira 13 (MEXU, UAMIZ); R. Lira 27 (ENCB, MEXU, UAMIZ, XAL); R. Ortega et al. 1090 (XAL); M. Palacios R. 30 (UAMIZ).

Cochlidium serrulatum (Sw.) L. E. Bishop

Atzalan: T. Krömer 3536 (XALU); S. Zamudio R. y P. Zamora 8469 (IEB). Tlalnelhuayocan: T. Krömer 3844 (XAL). San Andrés Tuxtla: T. Krömer y A. Acebey 2540 (XAL). Soteapan: R. Lira 14 (ENCB, UAMIZ, XAL). Zacualpan: C. A. Purpus 3021 (MO). 
Lellingeria delitescens (Maxon) A. R. Sm. \& R. C. Moran

(=Stenogrammitis delitescens (Maxon) Labiak)

San Andrés Tuxtla: T. Krömer 2561 (MEXU).

Lellingeria limula (H. Christ) A. R. Sm. \& R. C. Moran

(=Stenogrammitis limula (H. Christ) Labiak)

Pajapan: T. Krömer y E. Otto 2957 (MEXU).

Lellingeria prionodes (Mickel \& Beitel) A. R. Sm. \& R. C. Moran

(=Stenogrammitis prionodes (Mickel \& Beitel) Labiak)

Soteapan: M. Palacios R. 17 (UAMIZ). Mecayapan: T. Krömer y E. Otto 2441 (MEXU). San

Andrés Tuxtla: T. Krömer y A. Acebey 2561(MEXU).

Loxogramme mexicana (Fée) C. Chr.

La Perla: E. B. Copeland 156 (MEXU).

Melpomene leptostoma (Fée) A. R. Sm. \& R. C. Moran

Alpatláhuac: M. Nee y G. Schatz 19823 (XAL, XALU). Atzalan: T. Krömer et al. 3374 (MEXU); T. Krömer y J. Viccon E. 3466 (MEXU); F. Ventura A. 13336 (ENCB). Coscomatepec: M. Nee y G. Schatz 19832 (XALU). Ixhuacán de los Reyes: M. Cházaro B. y P. Padilla 3821 (IEB, XAL, XALU). Jalacingo: F. Ventura A. 341 (ENCB); F. Ventura A. 14523 (ENCB, XAL, XALU). San Andrés Tuxtla: T. Krömer 3929 (XALU). Tlalnelhuayocan: T. Krömer y J. Gómez D. 3949 (XAL, XALU). Zongolica: T. Krömer et al. 3138 (MEXU).

Melpomene pilosissima (M. Martens \& Galeotti) A. R. Sm. \& R. C. Moran

Acajete: C. Carvajal H. et al. CICH372 (XAL, XALU); C. Carvajal H. y S. Armenta M. CICH375 (XAL, XALU). Atzalan: T. Krömer y J. Viccon E.3537 (MEXU); T. Krömer et al. 3404 (MEXU).

Melpomene xiphopteroides (Liebm.) A. R. Sm.

Mecayapan: T. Krömer y E. Otto 2836 (MEXU). Tlalnelhuayocan: T. Krömer et al. 3851 (XAL, XALU); T. Krömer y J. Gómez D. 3938 (XAL, XALU). San Andrés Tuxtla: T. Krömer y A. Acebey 2078 (MEXU); T. Krömer y A. Acebey 2433 (MEXU, XAL); T. Krömer y A. Acebey 2543 (MEXU); T. Krömer et al. 2373 (MEXU). Soteapan: R. Riba et al. 1105 (UAMIZ). Tatahuicapan de Juárez: T. Krömer et al. 3665 (MEXU).

Microgramma lycopodioides (L.) Copel.

Catemaco: R. Riba et al. 1215 (MEXU, UAMIZ). Hidalgotitlán: M. Vázquez T. et al. 912 (XAL). Jesús Carranza: J. Dorantes 3913 (CORU, IEB, UAMIZ, XAL). Minatitlán: T. L. Wendt et al. 2596 (NY). Pajapan: R. Riba et al. 1189 (UAMIZ); R. Riba et al. 1198 (MEXU). Microgramma nitida (J. Sm.) A. R. Sm.

Actopan: R. Acosta P. 521 (XAL). Agua Dulce: A. D. L. Orozco S. 70 (MEXU). Atzalan: F. Ventura A. 17024 (ENCB, MEXU, XAL). Catemaco: J. H. Beaman 5669 (ENCB); J. I. Calzada 304 (MEXU); J. Calzada 364 (ENCB); A. Campos y C. León 5350 (MEXU); R. Cedillo T. 3429 (MEXU); P. Hietz y U. Seifert 859 (XAL); A. Mendoza R. et al. 1196 (IEB, UAMIZ); R. Riba 1935 (UAMIZ). Chicontepec: J. I. Calzada 5872 (XAL). Coatepec: G. Castillo C. y L. Tapia M. 1161 (XAL). Coatzacoalcos: J. I. Calzada (XAL); A. Mendoza R. et al. 143, 144, 145, (UAMIZ). Comapa: M. E. Medina A. y M. Ortiz D. (XAL). Ixhuatlán del Sureste: A. Mendoza R. 2074 (UAMIZ). Jalcomulco: G. Castillo C. y P. Zamora C. 8049 (XAL). Jesús Carranza: A. Mendoza R. et al. 1170, 1175 (IEB, UAMIZ). Las Choapas: A. Lot 1159 (MEXU); A. Mendoza R. et al 933 (IEB, UAMIZ). Martínez de la Torre: F. Ventura 
A. 50 (IEB); F. Ventura 13146 (ENCB, MEXU, XAL). Minatitlán: A. Mendoza R. et al. 146, 147 (UAMIZ). Naolinco: F. Ventura 13293 (ENCB, MEXU, XAL). Pajapan: L. Gutiérrez 21 (MEXU). Paso de Ovejas: M. E. Medina A. y M. Ortiz D. 710 (XAL). Poza Rica de Hidalgo: H. Sánchez y C. Chávez 562 (MEXU). Puente Nacional: M. Cházaro B. et al. 6111 (XAL). San Andrés Tuxtla: R. Cedillo T. 3805 (IEB, MEXU); R. Cruz C. 140 (ENCB, MEXU); T. Krömer et al. 2453 (MEXU); T. P. Ramamoorthy 3508 (MEXU); S. Sinaca C. y P. Ventura F. 1473 (UAMIZ). Soteapan: M. Leonti (MEXU). Tamiahua: J. Bonilla 874 (UAMIZ). Tampico Alto: G. Castillo C. et al. 25553 (UAMIZ, XAL). Texistepec: J. Chavelas P. et al. 27 (MEXU). Tlapacoyan: F. Ventura 723 (ENCB, MO). Uxpanapa: T. Krömer et al. 2678 (MEXU). Vega de Alatorre: B. Guerrero C. y J. I. Calzada 1900 (XAL). Sayula de Alemán: T. B. Croat 40021 (MEXU, MO).

Microgramma percussa (Cav.) de la Sota Hidalgotitlán: Brigada Dorantes 2765 (XAL); J. Dorantes 4081 (XAL); M. Vázquez 1038 (XAL); P. E. Valdivia Q. 1800 (XAL); P. E. Valdivia Q. 249, 345, 360, 387 (IEB, UAMIZ); P. E. Valdivia Q. s.n. (XAL); M. Vásquez et al. V-1038 (UAMIZ). Minatitlán: T. L. Wendt et al. 2634 (NY).

Microgramma piloselloides (L.) Copel.

Papantla: C. Landero M. 62 (XAL).

Micropolypodium trichomanoides (Sw.) A. R. Sm. (=Moranopteris trichomanoides (Sw.) R.Y. Hirai \& J. Prado)

Mecayapan: T. Krömer y E. Otto 2837 (MEXU). San Andrés Tuxtla: T. Krömer y A. Acebey 2541 (MEXU). Soteapan: R. Lira 28 (ENCB, MEXU, UAMIZ).

Niphidium crassifolium (L.) Lellinger

Atzalan: F. Ventura 87 (ENCB). Calcahualco: J. L. Martínez P. y F. Vázquez B. 279 (XAL). Camerino Z. Mendoza: T. B. Croat 39501(MO). Catemaco: A. Gómez-Pompa et al. 5360 (XAL); R. Lira 133 (UAMIZ); M. Nee y G. Schatz 19947 (MEXU). Coatepec: A. Espejo et al. 4432 (UAMIZ). Fortín: T. B. Croat 39431 (MO); T. Reeves et al. R-5898 (ASU); R. Riba 839 (MEXU). Hidalgotitlán: P. E. Valdivia Q. 2128 (XAL). Huatusco: J. I. Calzada 8037 (ENCB, MEXU); T. B. Croat 43976 (MO); E. R. de la Sota 4006 (ENCB). Huiloapan de Cuauhtémoc: J. I. Calzada 8560 (XAL). Ixtaczoquitlán: N. Flores E. 6 (CORU). Las Choapas: R. Riba y B. Pérez G. 1207 (UAMIZ). Rafael Delgado: A. Rincón G. y C. Durán E. 1411 (MEXU). Río Blanco: R. Torres C. y H. Hernández 3254 (MO, UAMIZ); E. Trujillo R. s.n. (CORU). San Andrés Tuxtla: R G. Ibarra M. et al. 2288 (UAMIZ); Riba 1939 (IEB, UAMIZ); S. Sinaca C. y F. Chigo S. 710 (UAMIZ); S. Sinaca C. et al. 957 (IEB, MO, UAMIZ). Soteapan: R. Acosta P. et al. 1627 (IEB); R. Lira 213 (ENCB, UAMIZ, XAL); A. Mendoza R. et al. 1187, 1192 (UAMIZ); R. Riba et al. 1126 (ENCB, MEXU, UAMIZ); R. Riba et al. 1166 (MEXU). Teocelo: F. Ventura 14049 (ENCB, MEXU, OAX). Tequila: P. E. Valdivia Q. 2184 (MO). Xico: A. Zlotnik 42 (UAMIZ). Zongolica: R. Riba 1312 (ENCB, UAMIZ); A. Rincón G. y C. Durán E. 1324 (MEXU). Zontecomatlán de López y Fuentes: A. Mendoza R. et al. 1657 (IEB, UAMIZ).

Pecluma alfredii (Rosenst.) M. G. Price

Acajete: F. Ventura 5324 (ENCB); F. Ventura A. 13711 (IEB). Acultzingo: E. R. de la Sota (ENCB). Camerino Z. Mendoza: T. B. Croat 39439, 39515 (MO). Chocamán: J. Rzedowski 19076 (ENCB). Coacoatzintla: F. Ventura 19365 (ENCB). Coscomatepec: E. R. de la Sota 
4055 (ENCB). Huatusco: J. Rzedowski 18930, 18952 (ENCB). Jalacingo: F. Ventura 42, 265 (ENCB). Orizaba: E. B. Copeland 127 (NY). San Andrés Tuxtla: T. Krömer y A. Acebey 1995A (MEXU). Zongolica: R. Riba 1310 (ENCB).

Pecluma alfredii (Rosenst.) M. G. Price var. cupreolepis (A. M. Evans) A. R. Sm.

Atzalan: F. Ventura 14556 (ENCB, IEB, OAX). Jilotepec: R. Riba et al. 956 (UAMIZ). Coacoatzintla: F. Ventura A. 19365 (MEXU).

Pecluma atra (A. M. Evans) M. G. Price

Atoyac: R. Acebedo 182 (IEB).

Pecluma consimilis (Mett.) M. G. Price

Catemaco: M. Nee y G. Schatz 19942 (MEXU). Minatitlán: T. Wendt y M. Ishiki I. 5881 (MEXU). San Andrés Tuxtla: J. H. Beaman y C. Álvarez del Castillo 5356 (MEXU).

Pecluma dispersa (A. M. Evans) M. G. Price

Hidalgotitlán: R. Riba y B. Pérez G. 889 (UAMIZ).

Pecluma divaricata (E. Fourn.) Mickel \& Beitel

Huatusco: F. Ventura A. 4952 (ENCB).

Pecluma plumula (Humb. \& Bonpl. ex Willd.) M. G. Price

Acajete: L. Sangabriel R. 56 (XALU). Jilotepec: R. Riba, B. Pérez y C. Vázquez 956 (ENCB). Pecluma ptilodon (Kunze) Lellinger

Atzalan: A. Ventura A. 533 (ENCB).

Pecluma sursumcurrens (Copel.) M. G. Price

Acajete: F. Ventura A. 5324 (MEXU). Atzalan: T. Krömer y J. Viccon E. 3547 (MEXU); T. Krömer et al. 3313 (MEXU). Banderilla: T. Krömer et al. 3754 (UAMIZ). Huatusco: S. Avendaño E. et al. 360 (MEXU). Huayacocotla: L. I. Nevling y A. Gómez-Pompa 1935 (MEXU). Huiloapan de Cuauhtémoc: S. Avendaño R. et al. 1049 (MEXU). Mecayapan: T. Krömer y E. Otto 2903, 2909 (MEXU). San Andrés Tuxtla: T. Krömer y A. Acebey 2067, 2082A (MEXU, XAL, XALU), 2195, 2496 (MEXU); T. Krömer, A. Acebey y A. Pérez P. 2322, 2391 (MEXU). Santiago Tuxtla: M. Sousa 2160 (MEXU). Tlalnelhuayocan: T. Krömer 3932 (XALU). Zongolica: T. Krömer et al. 3053 (MEXU).

Phlebodium areolatum (Humb. \& Bonpl. ex Willd.) J. Sm.

Acajete: J. L. Salazar R. 55 (XALU); L. Sangabriel R. 13, 34, 48 (XALU). Acula: F. Ventura A. 656 (NY). Amatlán de los Reyes: J. González L. 3 (CORU). Atzalan: T. Krömer et al. 3309 (UAMIZ); F. Ventura A. 17964 (ENCB, IEB). Banderilla: M. G. Zola B. 510 (ENCB, IEB); F. Ventura 13665 (ENCB). Calcahualco: J. L. Martínez P. y A. Espíritu 138 (IEB); J. L. Martínez P. y F. Vázquez B. 596 (IEB, MEXU). Chocamán: M. Nee 23240 (MEXU, NY). Coatepec: G. D. McPherson 970 (ENCB, NY). Coyutla: M. G. Zola B. 279 (NY). Fortín: E. B. Copeland 155 (NY). Huayacocotla: J. Viccon E. 220 (UAMIZ). Huiloapan de Cuauhtémoc, J. I. Calzada 8597 (XAL). Huatusco: E. R. de la Sota 4027 (ENCB); A. Mendoza R. et al. 1215 (IEB, UAMIZ). Ixhuacán de los Reyes: M. Nee 22949 (NY). Ixhuatlán del Café: A. Lot 867 (ENCB). Ixhuatlancillo: J. S. Miller y R. Torres C. 2988 (MEXU, MO). La Perla: F. Galicia G. 4 (CORU). Las Vigas de Ramírez: G. M. Diggs et al. 2116 (ENCB, NY). Naolinco: F. Ventura A. 8921 (ENCB, NY). Orizaba: A. Mendoza R. et al. 1163 (UAMIZ); L. Nevling y A. Gómez-Pompa 2281 (ENCB, MO). San Andrés Tuxtla: J. H. Beaman 5820 (MEXU); L. I. Nevling y A. Gómez-Pompa 2520 (ENCB, MEXU); F. Ventura A. 10230 (ENCB). San José de Gracia: F. Ventura 37 (ENCB). Soteapan: A. Mendoza R. et al. 1191 
(UAMIZ); R. Riba 1182 (UAMIZ). Teocelo: F. Ventura A. 13396 (ENCB); F. Ventura A. 16807 (IEB); M. G. Zola B. 142 (ENCB); M. G. Zola B. 156 (NY). Tepetzintla: G. Castillo C. et al. 2535 (MEXU). Tlalnelhuayocan: H. Narave F. y S. Avendaño R. 617 (XAL); M. Nee et al. 26170 (NY); P. Zamora C. 2922 (IEB); P. Zamora C. 2764 (MEXU). Tlapacoyan: M. Nee et al. 26086 (NY). Totutla: F. Ventura 16753, 10923 (ENCB). Xalapa: G. Carmona D. 45 (MEXU); Juan I. C. y Col. 2788 (UAMIZ). Xico: M. Nee y W. K. Taylor 29368 (NY). Yecuatla: C. Gutiérrez B. 3615 (IEB); Zongolica: A. Rincón G. y C. Durán E. 1319 (MEXU); P. E. Valdivia Q. 2126 (XAL); V. Vázquez 576 (XAL).

Phlebodium decumanum (Willd.) J. Sm.

Actopan: R. Acosta P. y N. Acosta B. 138 (XAL). Alto Lucero de Gutiérrez Barrios: J. Dorantes 5240 (XAL). Alvarado: J. Chavelas P. y C. Zamora S. s.n. (IEB, MEXU); A. Mendoza R. et al. 1197 (IEB, UAMIZ). Cosamaloapan de Carpio: A. Mendoza R. et al. 210 (UAMIZ). Juan Rodríguez Clara: A. Mendoza R. y B. Pérez G. 210 (UAMIZ). Papantla: H. Sánchez M. 633 (MEXU). Tecolutla: S. Zamudio R. s/n, (MEXU, UAMIZ). Tierra Blanca: A. Mendoza R. et al. 236 (UAMIZ).

Pleopeltis angusta Humb. \& Bonpl. ex Willd.

Acajete: J. L. Salazar R. 74 (XALU). Catemaco: A. Gómez-Pompa, W. et al. 5464 (UAMIZ); R. Lira 134 (UAMIZ). Chocamán: F. Ventura A. 3415 (ENCB); F. Ventura A. 16640 (ENCB, IEB, MEXU, MO). Coatepec: F. Ventura A. 9614 (ENCB). Fortín: T. Reeves et al. R-5878 (ASU); R. Riba 1314 (UAMIZ). Ixtaczoquitlán: S. Cortina V. 37 (ENCB, UAMIZ). Teocelo: V. Valdez T. 00058 (UAMIZ); F. Ventura A. 879 (ENCB). Xico: J. Ceja et al. 1194 (UAMIZ); B. Pérez G. et al. 37 (MEXU, UAMIZ); F. Ventura A. 11624 (ENCB, XAL).

Pleopeltis angusta Humb. \& Bonpl. ex Willd. var. angusta

Catemaco: Andrés Torres S. y A. Campos V. 19 (MEXU).

Pleopeltis angusta var. stenoloma (Fée) Farw.

Atoyac: R. Acevedo R. y R. Acosta P. 181 (MEXU, XAL). Calcahualco: J. L. Martínez P. y R. Acosta P. 1257 (XAL). Catemaco: R. Lira 156 (MEXU, UAMIZ); M. Nee y G. Schatz 19886 (XAL). Chocamán: M. Nee 23911 (XAL); R. Riba s.n. (MEXU). Coscomatepec: E. R. de la Sota 4041 (ENCB); F. Ventura A. 8729 (ENCB, MEXU); G. P. Villalobos y C. Martínez H. s.n. (CORU). Fortín: R. Flores M. 2 (CORU). Ixhuatlancillo: J. S. Miller y R. Torres C. 2984 (MEXU, MO). La Perla: O. Vignon O. 1 (CORU); A. Bozziere G. 1 (CORU). Nogales: M. Cid S. 31 (CORU). Orizaba: A. Mendoza R. et al. 1166 (IEB, UAMIZ); P. C. Mora G. 2 (CORU); S. H. Sohmer 9543 (MEXU). Río Blanco: P. Rossiles B. y H. Martínez R. 15 (CORU). Soteapan: R. Lira 235-A (MEXU, UAMIZ). Teocelo: D. S. Correll y H. B. Correll 28769 (MEXU). Tepatlaxco: Rzedowski 19051 (ENCB). Xico: F. Ventura A. 19208 (ENCB, IEB, MEXU, MO). Xalapa: A. Mendoza R. et al. 1686 (UAMIZ).

Pleopeltis astrolepis (Liebm.) E. Fourn.

Amatlán de los Reyes: J. Rzedowski 18871 (ENCB). Camerino Z. Mendoza: P. Rossiles B. y J. Carrera C. 9 (CORU). Catemaco: A. Mendoza R. et al. 862 (IEB, UAMIZ); T. P. Ramamoorthy et al. 3898 (MEXU); R. Riba y B. Pérez G. 1213 (ENCB, MEXU, UAMIZ); R. Riba 1570 (UAMIZ); R. Riba et al. 738 (MEXU, UAMIZ). Chiconquiaco: F. Ventura A. 8958 (ENCB). Jalacingo: F. Ventura A. 7609 (ENCB). Hidalgotitlán: P. E. Valdivia Q. 116, 801, 814, 1372, 1378 (IEB); P. E. Valdivia Q. 1171, 1337 (XAL); T. Wendt et al. 2738 (ENCB). Jesús Carranza: A. Mendoza R. et al. 1171 (UAMIZ). San Andrés Tuxtla: G. Ibarra M. 312 
(MO); T. P. Ramamoorthy 2608 (MEXU); S. Sinaca C. 418 (MEXU, UAMIZ). Santiago Tuxtla: J. J. den Held y F. A. van Rhijn HC3 (MEXU). Soteapan: R. Riba, B. Pérez G. y R. Lira 1140 (MEXU).

Pleopeltis crassinervata (Fée) T. Moore

Acajete: J. L. Salazar R. 31 (XALU). Acatlán: P. Hietz 291 (XAL). Atzalan: M. Nee y B. F. Hansen 18551 (MEXU); L. Zelaya, et al. 62 (UAMIZ). Calcahualco: J. L. Martínez P. y F. Vázquez B. 533 (XAL). Catemaco: A. Gómez-Pompa et al. 5434 (UAMIZ); R. Lira 112, 153 (MEXU, UAMIZ); R. Lira 158 (UAMIZ); R. Lira 181 (ENCB, UAMIZ, XAL). Chiconquiaco: R. Riba 623 (UAMIZ). Chocamán: R. Riba 842 (MEXU). Coatepec: R. Riba 672 (UAMIZ). Comapa: A. Mendoza R. et al. 1203 (UAMIZ). Huatusco: A. Mendoza R. et al. 1214 (UAMIZ). Jilotepec: F. Ventura A. 13656 (ENCB, IEB). Naolinco: F. Ventura A. 15757 (ENCB). San Andrés Tuxtla: T. Krömer y A. Acebey 1996, 2534 (MEXU); S. Sinaca C. et al. 984 (MEXU, MO, UAMIZ). Soteapan: R. Riba et al. 1105 (UAMIZ). Teocelo: D. S. Correll y H. B. Correll 28780 (MEXU); F. Ventura A. 18216 (MO). Tlacolulan: F. Ventura A. 9505 (XAL). Tlalnelhuayocan: P. Zamora C. 2934 (XAL). Tlapacoyan: F. Ventura A. 12534 (ENCB, IEB, MEXU). Totutla: C. Durán E. y T. Platas H. 1192 (MEXU, XAL). Xalapa: P. Weatherwax 169 (MO). Yecuatla: R. Riba 524 (UAMIZ); R. Riba 539 (ENCB, MEXU, UAMIZ). Zontecomatlán de López y Fuentes: A. Mendoza R. et al. 1649 (UAMIZ).

Pleopeltis fallax (Schltdl. \& Cham.) Mickel \& Beitel

Atzalan: J. I. Calzada 5214 (MEXU); T. Krömer et al. 3414 (UAMIZ); A. Mendoza R. et al. 1842 (UAMIZ); L. Pacheco et al. 3525 (UAMIZ); F. Ventura A. 18275 (ENCB, UAMIZ); F. Ventura A. 19487 (ENCB); F. Ventura 12226 (ENCB); L. Zelaya et al. 58 (UAMIZ). Catemaco: J. H. Beaman 5205 (MEXU); R. Lira 147 (MEXU, UAMIZ); R. Lira 175 (UAMIZ). Comapa: A. Mendoza R. et al. 1202 (IEB, UAMIZ). Hidalgotitlán: P. E. Valdivia Q. 984 (XAL). Huatusco: L. Pacheco 187 (IEB). San Andrés Tuxtla: T. Krömer y A. Acebey 1983 (MEXU, XAL); G. Ibarra M. y S. Sinaca C. 2942 (FCME, MEXU, UAMIZ); T. P. Ramamoorthy y G. Ibarra M. 3269 (MEXU); S. Sinaca C. 996 (UAMIZ). Teocelo: F. Ventura A. 18214 (MEXU). Tlapacoyan: M. E. Fraile O. et al. 60 (UAMIZ); F. Ventura A. 12226 (ENCB); F. Ventura A. 14868 (MEXU). Totutla: A. Espíritu y J. L. Martínez 167 (XAL); F. Ventura A. 5071 (ENCB); F. Ventura A. 17317 (MEXU, XAL). Misantla: D. S. Conant 764 (MEXU). Soteapan: R. Riba et al. 1086 (UAMIZ).

Pleopeltis mexicana (Fée) Mickel \& Beitel

Acajete: J. Dorantes et al. 1609 (UAMIZ); L. Sangabriel R. 166 (XALU); D. G. Saucedo 339 (ENCB); F. Ventura A. 5311 (ENCB, MEXU, XALU); F. Ventura A. 19390 (IEB). Acatlán: P. Hietz 96, 245 (XAL). Acultzingo: A. Lot 761 (MEXU); Nevling y A. Gómez-Pompa 2185 (UAMIZ); K. Roe et al. 1266 (ENCB). Atzalan: F. Ventura A. 11878 (IEB, MEXU). Banderilla: V. Hernández H. 02 (XALU). Chiconquiaco: F. Ventura A. 8958 (MEXU, XALU). Jalacingo: F. Ventura A. 7609 (MEXU). Las Vigas de Ramírez: L. Bohs et al. s. n. (MEXU). Xalapa: F. Ventura A. 9913 (IEB).

Pleopeltis polylepis (Roemer ex Kunze) T. Moore

Perote: H. Narave F. 879 (MEXU).

Pleopeltis polylepis (Roemer ex Kunze) T. Moore var. interjecta (Weath.) E. A. Hooper Banderilla: M. G. Zola B. 308 (MEXU). Huayacocotla: A. Mendoza R. et al. 1669 (UAMIZ). Xalapa: J. I. Calzada y Col. 2787 (UAMIZ); A. Mendoza R. et al. 1686 (UAMIZ). 
Pleopeltis polylepis (Roemer ex Kunze) T. Moore var. polylepis

Catemaco: R. Lira 116 (UAMIZ).

Pleopeltis x sordidula (Maxon \& Weath.) Mickel \& Beitel

Córdoba: J. H. Spence 114 (NY).

Polypodium alansmithii R. C. Moran

(=Pleopeltis alan-smithii (R. C. Moran) A. R. Sm. \& Tejero)

Yecuatla: C. Gutiérrez B. 3616 (MEXU).

Polypodium arcanum Maxon

Acajete: J. L. Salazar R. 71 (XALU); L. Sangabriel R. 36, 168 (XALU). La Perla: K. Montejo R. 2 (CORU). Orizaba: I. Rosas M. 1 (CORU); Y. Rivera S. 10 (CORU). Tlalnelhuayocán:

T. Krömer et al. 4002 (UAMIZ).

Polypodium bradeorum Rosenst.

(=Pleopeltis bradeorum (Rosenst.) A. R. Sm. \& Tejero)

Atoyac: E. B. Copeland 135 (NY).

Polypodium collinsii Maxon

(=Pleopeltis collinsii (Maxon) A. R. Sm. \& Tejero)

Catemaco: A. Gómez-Pompa et al. 5444 (XAL); R. Lira 202 (ENCB, MEXU); M. Nee y G. Schatz 19884 (MEXU). Mecayapan: T. Krömer y E. Otto 2888 (MEXU). San Andrés Tuxtla: J. I. Calzada 261 (ENCB, UAMIZ); R. Cedillo T. et al. 2983 (MEXU); T. Krömer et al. 2705 (MEXU); L. I. Nevling Jr. y A. Gómez-Pompa 2473 (ENCB, MO). Soteapan: J. I. Calzada 5096 (XAL). Yecuatla: C. I. Carvajal H. y S. Armenta M. 106 (MEXU); I. Carvajal H. et al. 116 (MEXU).

Polypodium colpodes Kunze

Orizaba: W. E. Manning y M. S. Manning 53751 (ENCB).

Polypodium conterminans Liebm.

Acajete: L. Sangabriel R. 61, 73, 114 (XALU). Atzalan: T. Krömer y J. Viccon E. 3502 (MEXU). Banderilla: T. Krömer et al. 3182, 3183 (MEXU). Jilotepec: F. Ventura A. 16831 (ENCB, IEB, MEXU). San Andrés Tuxtla: T. Krömer et al. 3624 (MEXU). Zongolica: T. Krömer et al. 3073 (MEXU).

Polypodium cryptocarpon Fée

(=Pleopeltis cryptocarpa (Fée) A. R. Sm. \& Tejero)

Atoyac: R. Acebedo y R. Acosta 174 (IEB). Catemaco: R. Lira 149 (MEXU, UAMIZ). Fortín: T. B. Croat 39424 (MO). Hidalgotitlán: J. Dorantes 3554 (IEB); T. Wendt et al. 2737 (ENCB, MEXU). Soteapan: R. Riba et al. 1180 (MEXU, UAMIZ).

Polypodium eatonii Baker

Banderilla: T. Krömer et al. 3743 (UAMIZ); T. Krömer et al. 3184 (MEXU); J. D. TejeroDíez y L. S. Sandoval 4315A, B, C, D (MO, UAMIZ); F. Ventura A. 13664 (IEB, MEXU). Ixhuacán de los Reyes: F. Ventura A. 16945 (IEB, MEXU, UAMIZ). Jilotepec: J. D. TejeroDíez y L. S. Sandoval 4317 (UAMIZ); F. Ventura A. 7110, 16825 (IEB, ENCB, MEXU); F. Ventura A. 10945 (MEXU, UAMIZ). Naolinco: J. I. Calzada 9400 (MEXU); F. Ventura A. 8918 (ENCB, MEXU, UAMIZ). Rafael Lucio: F. Ventura A. 649 (ENCB). Teocelo: D. S. Correll y H. B. Correll 28782 (MEXU); F. Ventura A. 12519 (ENCB, MEXU, UAMIZ). Xalapa: F. Ventura A. 16699 (IEB, MEXU, UAMIZ).

Polypodium echinolepis Fée 
Catemaco: R. Lira 139 (MEXU, UAMIZ); R. Lira 148 (UAMIZ). Coscomatepec: T. B. Croat y D. P. Hannon 63091 (MEXU). Huatusco: J. Rzedowski 18957 (ENCB). Jalacingo: F. Ventura A. 15148 (MEXU). Jilotepec: J. D. Tejero-Díez y L. S. Sandoval 4317 (MO). Las Minas: C. Durán E. y S. Avendaño R. 1129 (XAL). Córdoba: A. Sánchez V. 11 (CORU). Mecayapan: T. Krömer y E. Otto 2910 (MEXU). San Andrés Tuxtla: T. Krömer y A. Acebey 2484, 2707 (MEXU). Soteapan: J. Calzada 5147 (UAMIZ); M. Sousa 3558 (MEXU). Teocelo: D. S. Correll y H. B. Correll 28781 (MEXU); F. Ventura A. 12517 (ENCB); F. Ventura A. 13390, (ENCB XAL); F. Ventura A. 15797 (ENCB, MEXU, MO); F. Ventura A. 16812 (ENCB, IEB, MEXU); F. Ventura A. 18219 (ENCB). Xalapa: C. G. Pringle 11855 (MO). Xico: F. Ventura A. 19210 (ENCB). Zongolica: R. Riba 1292 (ENCB).

Polypodium fraternum Schltdl. \& Cham.

Acula: F. Ventura A. 10371 (ASU, ENCB, MEXU). Calcahualco: J. L. Martínez P. 2015 (IEB). Miahuatlán: F. Ventura A. 14629 (IEB, MEXU). Naolinco: F. Ventura A. 12199 (IEB, MEXU). Soteapan: R. Riba et al. 1153 (MEXU). Tlaltetela: R. T. Clausen s. n. (MEXU).

Polypodium furfuraceum Schltdl. \& Cham.

(=Pleopeltis furfuracea (Schltdl. \& Cham.) A. R. Sm. \& Tejero)

Acajete: J. L. Salazar R. 54 (XALU); L. Sangabriel R. 158 (XALU); F. Ventura A. 12751 (ENCB, MEXU). Acula: F. Ventura A. 10840 (ENCB, IEB). Amatlán de los Reyes: S. Castellano M. 6 (CORU); M. R. Pérez T. 1 (CORU); J. Rzedowski 750 (ENCB). Atzalan: M. Nee y B. F. Hansen 18570 (MEXU); M. Nee y B. F. Hansen 18699 (MEXU, XAL); F. Ventura A. 15, 156 (ENCB). Banderilla: T. Krömer et al. 3739 (UAMIZ). Calcahualco: J. L. Martínez P. y F. Vázquez 210, 700 (IEB, MEXU); J. R. Muñoz P. 5 (CORU). Catemaco: J. H. Beaman 6442 (UAMIZ); R. Riba et al. 737 (MEXU). A. Torres S. y A. Campos V. 20 (MEXU). Chocamán: R. Riba (MEXU); J. Rzedowski 1350 (ENCB); J. Rzedowski 19077 (ENCB, MEXU). Coatepec: T. Duncan 2444 (ENCB); F. Ventura A. 10381 (ASU, ENCB, IEB, MEXU). Comapa: A. Mendoza R. et al. 1200 (UAMIZ). Córdoba: T. S. Elias et al. 1114 (MO). Coscomatepec: T. B. Croat 43949 (MO); T. B. Croat 43966 (MEXU, MO); M. E. Murrieta G. 13 (CORU). Fortín: T. B. Croat 39432 (MO); G. Ramírez et al. s.n. (CORU). Hidalgotitlán: Brigada Dorantes 2546 (MEXU, XAL); P. E. Valdivia Q. 228, 1159 (IEB). Huatusco: T. B. Croat 43994 (MO); E. R. de la Sota 4007 (ENCB); A. Mendoza R. et al. 1218 (UAMIZ). Ixhuatlán del Café: F. Ventura A. 16258 (ENCB, MEXU, XAL). Ixtaczoquitlán: A. Torres R. 25 (CORU); E. Marcos N. 17 (CORU). Jalcomulco: G. Castillo C. y P. Zamora C. 7594 (XAL). Jilotepec: L. Bohs et al. 1650 (MEXU). Minatitlán: T. L. Wendt y M. Ishiki I. 5882 (MO). Orizaba: C. H. Ávila B. 6 (MEXU); S. Canizales Q. y J. Elvir E. 2 (CORU); J. González V. 11 (ENCB); W. E. Manning y M. S. Manning 53755 (MEXU); A. Mendoza R. et al. 1167 (IEB, UAMIZ); K. Reyes L. 5 (CORU). Río Blanco: R. Torres C. y H. Hernández 3258 (MEXU). Soteapan: A. Mendoza R. et al. 1194 (UAMIZ). Teocelo: D. S. Correll y H. B. Correll 28779 (MEXU); R. Riba 673 (UAMIZ). Tlacotepec de Mejía: C. A. Purpus s. n. (MO). Xalapa: J. G. García F. y J. Palma G. 46 (MEXU, XAL); M. Nee 23548 (MEXU); F. Ventura A. 2392 (ENCB); F. Ventura A. 9893 (ASU, ENCB, MEXU); F. Ventura A. 10191 (IEB). Zongolica: González-Vidal et al. 4 (CORU).

Polypodium hartwegianum Hook.

Calcahualco: J. L. Martínez P. y F. Vázquez 343 (IEB). Naolinco: J. I. Calzada 9402 (IEB, $\mathrm{XAL}$ ). 
Polypodium hispidulum Bartlett

Santiago Tuxtla: M. Sousa 2153 (MEXU); L. Zelaya et al. 77 (UAMIZ). Soteapan: R. Riba et al. 1148-A (UAMIZ).

Polypodium lepidotrichum (Fée) Maxon

(=Pleopeltis lepidotricha (Fée) A. R. Sm. \& Tejero)

Acula: F. Ventura A. 10280 (ASU, IEB). Atzalan: F. Ventura A. 8435 (ENCB, MEXU, XALU). Chiconquiaco: F. Ventura A. 8281 (ENCB, MEXU); F. Zavala C. 294 (MEXU). Coscomatepec: E. R. de la Sota 4057 (ENCB); F. Ventura A. 4901 (ENCB, MEXU, UAMIZ). Huatusco: E. R. de la Sota 4002 (ENCB); J. Rzedowski 18980 (ENCB). Huayacocotla: L. Ballesteros y F. Ballesteros 467 (IEB, MEXU, XAL); A. Mendoza R. et al. 1666 (IEB, UAMIZ). Jalacingo: F. Ventura A. 13084 (UAMIZ). Misantla: D. S. Barrington y D. S. Conant 768 (MEXU). Orizaba: C. G. Pringle s.n. (MO). Xalapa: G. Castillo C. et al. 48 (XAL); J. Dorantes y M. Acosta 2096 (ENCB).

Polypodium liebmannii C. Chr.

Acajete: F. Ventura A. 201 (ENCB); F. Ventura A. 4672 (ENCB, MEXU, UAMIZ); F. Ventura A. 5604 (ENCB, MEXU, UAMIZ). Chiconquiaco: F. Ventura A. 11703 (ENCB, IEB).

Polypodium longepinnulatum E. Fourn.

Acajete: J. L. Salazar R. 61 (XALU); L. Sangabriel R. 108 (XALU). Catemaco: R. Lira 122 (UAMIZ); R. Lira 127 (MEXU, UAMIZ). Coscomatepec: E. R. de la Sota 4056 (ENCB); A. Lot 1178 (MEXU, MO). Orizaba: E. B. Copeland 130 (MEXU); C. G. Pringle 5953 (MO). Tlalnelhuayocan: F. Ventura A. 16620 (ENCB, IEB, MEXU). Xalapa: J. I. Calzada 1938 (IEB, MEXU, XAL); A. Ventura A. 10687 (ASU, ENCB). Xico: P. Tenorio L. 15547 (MEXU). Tlacotepec de Mejía: L. I. Nevling y A. Gómez-Pompa 2445 (ENCB).

Polypodium madrense J. Sm.

(=Pleopeltis madrensis (J. S.) A. R. Sm. \& Tejero)

Acajete: L. Sangabriel R. 16 (XALU). Acatlán: F. Ventura A. 15782 (ENCB). Acula: F. Ventura A. 10281 (ENCB, IEB, MO). Acultzingo: N. Martínez Correa et al. 21 (UAMIZ). Atzalan: F. Ventura A. 11877 (ENCB, IEB).

Polypodium martensii Mett.

Las Vigas de Ramírez: F. Ventura A. 7550 (ENCB, XALU).

Polypodium plebeium Schldtl. \& Cham.

(=Pleopeltis plebeia (Schltdl. \& Cham.) A.R. Sm. \& Tejero)

Acajete: J. L. Salazar R. 53 (XALU); L. Sangabriel R. 180 (XALU); F. Ventura A. 5312 (ENCB). Acatlán: F. Ventura A. 15782 (IEB, MEXU). Altotonga: A. Carrillo S. s/n (ENCB, MO, UAMIZ). Atzalan: L. Pacheco et al. 3523 (UAMIZ); F. Ventura A. 1530 (UAMIZ); F. Ventura A. 11877, 12612 (MEXU). Ayahualulco: M. Nee 22892 (MEXU). Banderilla: A. E. Del Ribero M. 100 (XALU). Calcahualco: J. L. Martínez 8 (MEXU); J. L. Martínez P. y F. Vázquez B. 212 (XAL); J. L. Martínez y F. Vázquez 541 (MEXU). Catemaco: R. Lira 179 (MEXU, UAMIZ). Chiconquiaco: M. Palacios R. 3290 (XAL). Córdoba: A. Sánchez V. 9 (CORU); A. Sánchez V. 8 (CORU). Coscomatepec: T. B. Croat 100085 (MO). Huatusco: E. R. de la Sota 4001, 4025 (ENCB); A. Mendoza R. et al. 1174 (UAMIZ). Huayacocotla: R. Hernández M. 1456 (XAL); A. Mendoza R. et al. 1663 (UAMIZ). Ixhuatlancillo: J. Miranda B. 12 (CORU). Jalacingo: L. I. Nevling y A. Gómez-Pompa 1067 (MEXU); F. Ventura 41, 127, 
128 (ENCB); F. Ventura A. 7606 (XALU); F. Ventura A. 7607, 13081 (ENCB, IEB, MEXU); F. Ventura A. 18576 (MEXU). La Perla: F. Galicia G. 3 (CORU); A. Romero G. 1 (CORU); M. Ávila V. 2 (CORU). Las Vigas de Ramírez: J. Dorantes 565 (ENCB); R. Ortega O. s.n. (XALU); M. Palacios R. 3270 (XAL). Los Reyes: L. García et al. s.n. (FCME). Miahuatlán: F. Ventura 11830 (ENCB, UAMIZ). Misantla: D. S. Conant 762 (MEXU). Naolinco: F. Ventura A. 15059 (IEB, ENCB, MEXU). Orizaba: E. B. Copeland 1366 (MEXU); A. Mendoza R. et al. 1168 (UAMIZ). Rafael Lucio: F. Ventura A. 101281 (MEXU). San Andrés Tuxtla: R. Cedillo T. et al. 2987 (FCME, MEXU); T. B. Croat y P. Díaz J. 100342 (MO); L. I. Nevling y A. Gómez-Pompa 2471 (ENCB, MO); L. I. Nevling y A. Gómez-Pompa 2472 (ENCB, MEXU). San Juan Tepeuxila: R. Torres C. et al. 16230 (MEXU). Soteapan: R. Lira 234 (MEXU, UAMIZ). Tepetzintla: G. Castillo C. y A. Benavides M. 2194 (MEXU). Xalapa: J. I. Calzada 2784 (UAMIZ); E. Martínez V. et al. 65 (UAMIZ). Yecuatla: J. I. Calzada 4714 (XAL); A. Gómez-Pompa y R. Riba 344 (UAMIZ); R. Riba 906 (MEXU, UAMIZ). Zongolica: A. Rincón G. y C. Durán E. 1452 (MEXU). Zontecomatlán de López y Fuentes: A. Mendoza R. et al. 1649 (UAMIZ).

\section{Polypodium plesiosorum Kunze}

Acajete: R. Riba 1613 (IEB); F. Ventura A. 12750 (IEB, MEXU); F. Ventura A. 19580 (MO). Acatlán: F. Ventura A. 10750 (ASU, ENCB, MO). Acultzingo: E. R. de la Sota 1132 (ENCB). Banderilla: W. Márquez R. 487 (IEB, MEXU). Calcahualco: M. Nee y G. Schatz 19706 (MEXU). Camerino Z. Mendoza: T. B. Croat 39500 (MO). Catemaco: R. Lira 110, 157 (UAMIZ). Fortín: E. B. Copeland 131a (MEXU). Jalacingo: F. Ventura A. 18576 (ENCB). Santiago Tuxtla: M. Sousa 3195 (MEXU). Soteapan: R. Riba et al. 1132 (ENCB, MEXU). Xalapa: M. de la Rosa 6 (IEB). Zongolica: R. Riba 1301 (ENCB).

Polypodium pleurosorum Kunze ex Mett.

Xico: F. Ventura A. 19210 (MEXU). Zongolica: R. Riba 1292 (MEXU).

Polypodium polypodioides (L.) Watt

(=Pleopeltis polypodioides (L.) E. G. Andrews \& Windham)

Altotonga: F. Ventura A. 63 (ENCB). Atoyac: R. Acevedo R. y G. Castillo C. 232 (MEXU). Amatlán de los Reyes: R. Alvizar D. 1 (CORU). Atzalan: T. Krömer et al. 3406 (UAMIZ); R. Ortega O. 1847 (UAMIZ); L. Pacheco et al. 3524 (UAMIZ); F. Ventura A. 8344 (MEXU, XALU). Banderilla: A. M. Aquino Z. 87 (XALU). Catemaco: R. L. Dressler y Q. Jones 96 (MEXU); R. Lira 144 (MEXU, UAMIZ); R. Lira 150 (MEXU, XAL); R. Lira 174 (MEXU, UAMIZ); F. Menéndez L. 31 (MO); D. Ortíz y M. González s.n. (UAMIZ); R. Riba 1932 (UAMIZ); M. Sousa 2554 (MEXU). Córdoba: C. R. Orcutt 3374 (MEXU). Coscomatepec: T. B. Croat 43949a (MO). Emiliano Zapata: P. Hietz 17 (XAL). Hidalgotitlán: P. E. Valdivia Q. 568 (XAL). San Andrés Tuxtla: J. I. Calzada 1546 (UAMIZ); G. Ibarra M. et al. 2154 (MEXU, UAMIZ). Soteapan: R. Riba et al. 1147 (UAMIZ); R. Riba y R. Lira 1179 (MEXU, UAMIZ). Tantima: R. Riba 1327 (UAMIZ). Tempoal: M. Nee y B. F. Hansen 18381 (XAL). Tlaltetela: R. T. Clausen (MEXU). Tlapacoyan: F. Ventura A. 12533 (MEXU, UAMIZ).

Polypodium polypodioides (L.) Watt var. aciculare Weath.

Acajete: J. L. Salazar R. 70 (XALU). Ixhuacán de los Reyes: A. Smith P. 13 (XALU).

Polypodium polypodioides (L.) Watt var. polypodioides

Chinameca: A. Mendoza R. et al. 1183 (UAMIZ). Comapa: A. Mendoza R. et al. 1199 (UAMIZ). Jesús Carranza: A. Mendoza R. et al. 1172 (UAMIZ). Sayula de Alemán: A. 
Mendoza R. et al. 1169 (UAMIZ). Texistepec: C. H. Ramos y E. Martínez S. 6, 1050 (MEXU). Tonayán: S. Avendaño R. 9 (MEXU).

Polypodium polypodioides var. michauxianum Weath.

Catemaco: R. Riba y B. Pérez G. 1214 (ENCB, MEXU, UAMIZ). Soteapan: R. Ortega et al. 1050 UAMIZ). Teocelo: V. Vázquez T. 86 (UAMIZ).

Polypodium puberulum Schltdl. \& Cham.

Altotonga: F. Ventura 111, 358 (ENCB). Banderilla: W. Márquez R. 504 (MEXU, XAL). Chiconquiaco: F. Ventura 8581 (ENCB, UAMIZ). Huatusco: A. Mendoza R. et al. 1210 (IEB, UAMIZ).

Polypodium pyrrholepis (Fée) Maxon

(=Pleopeltis pyrrholepis (Fée) A. R. Sm. \& Tejero)

Atoyac: R. Acevedo R. y R. Acosta 185 (IEB); E. B. Copeland 141 (MEXU). Comapa: A. Mendoza R. et al. 1201 (UAMIZ). Hidalgotitlán: Brigada Dorantes 2547 (MEXU); T. Wendt y A. Villalobos C. 3968 (ENCB, MEXU). Huatusco: E. R. de la Sota 4023, 4040 (ENCB); J. Rzedowski 18912 (ENCB). Minatitlán: T. Wendt et al. 2928 (MEXU, XAL). Totutla: F. Ventura A. 13542 (IEB, MEXU, XAL).

Polypodium remotum Desv.

Acajete: L. Sangabriel R. 55 (XALU). Xico: J. I. Calzada 8339 (XAL).

Polypodium rhachipterygium Liebm.

Catemaco: A. Gómez P. et al. 5463 (UAMIZ, XAL); R. Riba et al. 1214-A (ENCB, MEXU, UAMIZ). Hidalgotitlán: R. Riba y B. Pérez 800 (XAL). Jesús Carranza: M. Vázquez T. et al. V-2487 (MEXU). San Andrés Tuxtla: J. I. Calzada 1539 (FCME); M. Sousa 2093 (MEXU). Polypodium rhodopleuron Kunze

Acajete: J. L. Salazar R. 81 (XALU). Acula: F. Ventura 2223 (ENCB). Atzalan: S. H. Sohmer 9511 (MEXU). Banderilla: J. D. Tejero-Díez y L. S. Sandoval 4316 (MO). Chiconquiaco: F. Ventura A. 12270 (ENCB, MEXU). Huatusco: A. Mendoza R. et al. 1216 (IEB, UAMIZ); M. Winkler s. n. (XAL). Ixhuacán de los Reyes: M. Chazaro B. y P. Padilla 3807 (IEB). Jilotepec: M. Winkler s. n (XAL). Miahuatlán: F. Ventura A. 11833 (ENCB, MEXU, UAMIZ). Naolinco: F. Ventura A. 12198 (ASU, MO); F. Ventura A. 16804 (ASU, MO). Nautla: J. D. Dwyer 14384 (MEXU, MO). Soteapan: R. Acosta P. y L. Ceja 1674 (IEB); A. Mendoza R. et al. 1195 (UAMIZ). Tepetzintla: G. Castillo C., M. Nee y A. Benavides M. 2502 (IEB, MEXU). Tlachichilco: A. Rincón G. et al. 2559 (XAL). Xalapa: J. I. Calzada 1905, 1933 (MEXU); G. Castillo et al. 51 (XAL); D. S. Correll y H. B. Correll 28762 (MEXU); K. Fabián et al. 387 (XAL); S. Scheffknecht 29 (XAL). Xico: P. Hietz y U. Seifert 811 (XAL). Zongolica: E. Lazos et al. s.n. (FCME).

Polypodium rzedowskianum Mickel

(=Pleopeltis rzedowskiana (Mickel) A. R. Sm. \& Tejero)

San Andrés Tuxtla: M. Sousa 3447 (MEXU).

Polypodium sanctae-rosae (Maxon) C. Chr.

(=Pleopeltis sancatae-rosae (Maxon) A. R. Sm. \& Tejero)

Catemaco: T. P. Ramamoorthy et al. 3900 (MEXU).

Polypodium subpetiolatum Hook.

Jalacingo: F. Ventura A. 15148 (ENCB, IEB). Chocamán: M. Nee 23242 (MEXU).

Teocelo: F. Ventura A. 12517, 14830 (IEB, MEXU). 
Polypodium villagranii Copel.

(=Pleopeltis villagranii (Copel.) A. R. Sm. \& Tejero)

Atzalan: T. Krömer et al. 3451 (MEXU). Ixhuacán de los Reyes: M. Nee 22495 (XAL). Zontecomatlán de López y Fuentes: A. Mendoza R. et al. 1648 (UAMIZ).

Serpocaulon falcaria (Kunze) A. R. Sm.

Acajete: J. L. Salazar R. 68 (XALU); L. Sangabriel R. 005, 59 (XALU). Atzalan: T. Krömer 3344 (MEXU). Huatusco: F. Ventura A. 4800 (ENCB). Tlalnelhuayocan: C. Gallardo H. y L. Lozada 3174 (XAL) T. Krömer et al. 3924 (XALU). Soteapan: R. Lira 17 (UAMIZ). Zongolica: T. Krömer et al. 3072 (MEXU).

Serpocaulon fraxinifolium (Jacq.) A. R. Sm.

Catemaco: R. Riba y B. Pérez G. 1211 (MEXU).

Serpocaulon loriceum (L.) A. R. Sm.

Acajete: F. Ventura A. 4668 (ENCB, MEXU). Atzalan: F. Ventura A. 11111 (IEB, MEXU); F. Ventura A. 14369 (MEXU, XAL); L. Zelaya et al. 66 (UAMIZ). Chiconquiaco: A. P. Vovides 291 (XAL). Coscomatepec: F. Ventura A. 4907 (ENCB). Huatusco: F. Ventura A. 7737 (ENCB); F. Ventura A. 13126 (IEB, XAL). Juchique de Ferrer: G. Castillo C. et al. 1709 (IEB, XAL). Los Reyes: A. Rincón G. y C. Durán E. 1392 (MEXU). Misantla: L. Bohs et al. 1813 (MEXU). Soteapan: R. Lira 12 (MEXU); M. Palacios R. 21 (UAMIZ); R. Riba et al. 1089 (MEXU). Xico: M. Nee y K. Taylor 26312 (XAL). Yecuatla: R. Riba 535 (UAMIZ). Serpocaulon triseriale (Sw.) A. R. Sm.

Amatlán de los reyes: R. Alvizar D. 4 (CORU). Atzalan: F. Ventura A. 9482 (ENCB); F. Ventura A. 12506 (MEXU). Catemaco: J. H. Beaman 6470 (MEXU, UAMIZ); R. Cedillo T. 3912 (MEXU, UAMIZ); F. Menéndez L. 28 (ASU); F. Menéndez L. 113 (MEXU); R. Riba 1933 (IEB, UAMIZ); R. Riba 1694, 1938 (UAMIZ); D. Tejero D. 4186 (UAMIZ); R. H. Warner 385 (MEXU). Coatepec: J. I. Calzada y F. Vázquez 10227 (XAL); P. Hietz 264 (XAL). Comapa: A. Mendoza R. et al. 1198 (IEB, UAMIZ). Córdoba: C. M. Rowell Jr. et al. 17M6 (MEXU). Hidalgotitlán: P. E. Valdivia Q. 151, 1328 (XAL); T. L. Wendt y A. Villalobos C. 3965 (NY). Huatusco: T. B. Croat 39636 (MEXU); F. Ventura A. 7141 (ENCB). Jalacingo: F. Ventura A. 12216 (ENCB, MEXU, XAL). Jalcomulco: G. Castillo y P. Zamora 7597 (XAL). Jesús Carranza: A. Mendoza R. et al. 1174 (IEB, UAMIZ); M. Vázquez et al. 2580 (IEB, MEXU). Las Choapas: A. Franco M. et al. 99 (IEB, XAL). Meyacapan: T. Krömer y E. Otto 2876 (XAL). Pajapan: M. Nee y J. I. Calzada 22749 (XAL). San Andrés Tuxtla: J. I. Calzada 1571 (MEXU, OAX); G. Ibarra M. 936 (MEXU); G. Ibarra M. et al. 3028 (MEXU, UAMIZ); T. P. Ramamoorthy 2637 (MEXU). Soteapan: R. Riba et al. 1127 (ENCB, MEXU, UAMIZ); R. Riba et al. 1150, 1172, 1181 (MEXU, UAMIZ). Tezonapa: E. García M. 1 (CORU). Tlapacoyan: F. Ventura A. 19609 (ENCB, MEXU, XAL); F. Ventura A. 20345 (ASU). Uxpanapa: T. Krömer et al. 2673, 2676 (MEXU). Xico: F. Vázquez B. 517 (XAL).

Terpsichore asplenifolia (L.) A. R. Sm.

Atzalan: T. Krömer 3472 (MEXU, UAMIZ); F. Ventura A. 11610 (ASU, IEB, MEXU, MO, XAL, XALU); F. Ventura A. 15225 (ASU, MO). Coatepec: T. Krömer 3883 (XAL, XALU). Huatusco: J. González V. 25 (UAMIZ, XAL); M. L. Monge et al. 98 (XALU); F. Ventura A. 13127 (ENCB, MO). Tlalnelhuayocan: C. Carvajal H. et al. 408 (XAL, XALU). Soteapan: R. Lira 231 (UAMIZ). Teocelo: F. Lorea 381 (FCME). Totutla: F. Ventura A. 1200 (MEXU); 
F. Ventura A. 5119 (ENCB); F. Ventura A. 11314 (ASU, MO); F. Ventura A. 16752 (IEB). Xalapa: C. G. Pringle 8158 (IEB, MEXU, MO).

Terpsichore cultrata (Willd.) A. R. Sm.

(=Alansmia cultrata Moguel \& M. Kessler)

Tlalnelhuayocan: C. Carvajal H. et al. 326 (XAL, XALU).

Terpsichore mollissima (Fée) A. R. Sm.

(=Alansmia elastica (Bory ex Willd.) Moguel \& M. Kessler)

San Andrés Tuxtla: T. Krömer 2164 (MEXU, XAL).

\section{PSILOTACEAE}

Psilotum complanatum Sw.

Acajete: J. L. Salazar R. 45 (XALU); L. Sangabriel R. 25 (XALU); F. Ventura 13326 (ENCB, MO). Atzalan: S. D. Koch y E. E. Terrell 8754 (XAL, XALU); T. Krömer 3314 (MEXU); S. Zamudio R. y P. Zamora 8467 (MEXU, UAMIZ); S. Zamudio R. y P. Zamora 8468 (IEB, MEXU, UAMIZ). Chiconquiaco: G. Cortes R. s. n. (CORU). Chocamán: J. A. Vázquez y O. Sánchez E. 8 (CORU). Coatepec: R. Ortega O. et al. 2546 (XALU). Ixhuacán de los Reyes: M. Cházaro B. y P. Padilla 3725 (XAL, XALU); A. E. del Ribero M. 15 (XALU); P. Hietz y U. Seifert 802 (XAL). Ixhuatlancillo: O. M. Illescas S. (CORU). Misantla: A. Gómez P. 1569 (XAL). Rafael Lucio: H. Oliva et al. 1596 (CORU). Tlalnelhuayocan: A. Flores-Palacios y J. G. García-Franco 442, 928, 891 (XAL, XALU); J. Gómez-Díaz et al. 87 (XALU). Yecuatla: J. I. Calzada 4309 (XAL); A. Mendoza R. y J. Ceja 256 (UAMIZ); A. P. Vovides y R. Riba 295 (XAL). Zongolica: H. Flores M. 38 (CORU); T. Krömer 3058 (MEXU).

Psilotum nudum (L.) P. Beauv.

Catemaco: A. Lot 1498 (MEXU).

\section{PTERIDACEAE}

Ananthacorus angustifolius (Sw.) Underw \& Maxon

Hidalgotitlán: Brigada Dorantes 2767 (XAL); P. E. Valdivia Q. 491, 1706 (XAL). Las Choapas: E. López P. 616 (XAL); A. Rincón G. et al. 3055 (XAL). Soteapan: R. Riba et al. 1141 (MEXU, UAMIZ); R. Riba et al. 1149 (UAMIZ).

Anetium citrifolium (L.) Splitg.

Las Choapas: A. Franco M. et al 97 (XAL); A. Mendoza R. et al. 906 (UAMIZ). Minatitlán: T. Wendt et al. 5802 (MEXU, NY). Tlapacoyan: F. Ventura 12429 (MEXU).

Polytaenium cajenense (Desv.) Benedict

Hidalgotitlán: P. E. Valdivia Q. 1826 (XAL).

Polytaenium feei (W. Schaffn. ex Fée) Maxon

Catemaco: R. Lira 171 (UAMIZ); R. Lira 172-b (XAL); R. Riba et al. 1193 (UAMIZ). Hidalgotitlán: R. Riba y B. Pérez G. 817 (XAL). Misantla: D. S. Conant 783 (MEXU). San Andrés Tuxtla: T. Krömer et al. 1926 (MEXU); T. Krömer y A. Acebey 1993 (MEXU); T. Krömer et al. 2332 (MEXU); A. Pérez P. et al. 7 (MEXU). Tlacotepec de Mejía: C. A. Purpus 2230 (MO).

Polytaenium lineatum (Sw.) J. Sm. Hidalgotitlán: P. E. Valdivia Q. 733, 1286 (XAL); P. E. Valdivia Q. et al. 1349 (XAL). San Andrés Tuxtla: T. Krömer 2368 (MEXU). 
Scoliosorus ensiformis (Hook.) T. Moore

Atzalan: T. Krömer 3441 (MEXU). Catemaco: J. H. Beaman 6154 (UAMIZ). Mecayapan: T. Krömer y E. Otto 3003 (MEXU). Pajapan: J. I. Calzada 425 (MO). Santiago Tuxtla: J. H. Beaman y Álvarez 5767 (UAMIZ). San Andrés Tuxtla: R. L. Dressler y Q. Jones 147 (MO); G. Diggs et al. 2681 (XAL); T. Krömer y A. Acebey 1994 (MEXU, XAL); T. Krömer et al. 2321 (MEXU); T. Krömer et al. 3622 (MEXU, UAMIZ); A. Pérez P. et al. 6 (CORU, MEXU); A. Rincón et al. 2426 (MEXU, XAL). Soteapan: R. Lira 220 (UAMIZ); M. Palacios R. 24 (UAMIZ, XAL); R. Riba et al. 1117 (UAMIZ). Tlalnelhuayocan: T. Krömer et al.3916 (XALU). Tlapacoyan: F. Ventura A. 12429 (MEXU). Totutla: F. Ventura A. 8212 (IEB, ASU). Xico: H. Narave F. 345 (IEB, XAL); H. Narave F. 62092 (MEXU).

Vittaria bradeorum Rosenst.

San Andrés Tuxtla: T. Krömer et al. 3621 (MEXU). Zongolica: H. Flores M. 63 (CORU); T. Krömer et al. 3226 (CORU, MEXU).

Vittaria flavicosta Mickel \& Beitel

Catemaco: J. H. Beaman 6135 (MEXU). Hidalgotitlán: P. E. Valdivia Q. 121, 1530, 1819 (XAL); M. Vázquez 59 (XAL). Mecayapan: M. Nee et al. 25019 (MEXU). Pajapan: T. Krömer y E. Otto 2961 (MEXU). San Andrés Tuxtla: T. Krömer y A. Acebey 1992 (MEXU, XAL); T. Krömer y A. Pérez P. 2775 (MEXU); T. Krömer et al. 1960 (MEXU); T. Krömer et al. 2330 (MEXU); T. Krömer et al. 2390 (MEXU); A. Pérez P. et al. 5 (CORU, MEXU). Soteapan: T. Krömer et al. 3587 (MEXU). Xalapa: P. Hietz 50 (XAL).

Vittaria graminifolia Kaulf.

Acajete: R. Riba 1945 (UAMIZ); J. L. Salazar R. 32 (XALU); F. Ventura A. 18166 (MEXU, UAMIZ, XAL). Atzalan: T. Krömer y J. Viccon E. 3546 (MEXU); T. Krömer et al. 3376 (MEXU). Banderilla: T. Krömer et al. 3186 (MEXU, UAMIZ). Catemaco: A. Gómez-Pompa et al. 5362 (UAMIZ); R. Lira 172 (UAMIZ). Coatepec: M. Cházaro y L E. Castillo 6788 (XAL). Coscomatepec: T. B. Croat y D. P. Hannon 63106 (MEXU, MO); E. R. de la Sota 7920 (ENCB). Chiconquiaco: J. I. Calzada 9342 (IEB, XAL); I. Pérez G. y L. Mendizábal 156 (MEXU); F. Ventura 7920 (ENCB, XALU). Hidalgotitlán: M. Vázquez et al. 59 (ENCB). Huatusco: A. Mendoza R. et al. 1208 (UAMIZ). Mecayapan: T. Krömer y E. Otto 3004 (MEXU). San Andrés Tuxtla: T. Krömer y A. Acebey 2483 (MEXU); G. Ibarra M. et al. 3013 (UAMIZ). Soteapan: T. Krömer et al. 3621 (MEXU); R. Lira 25 (UAMIZ); R. Ortega O. 1087 (IEB, UAMIZ); R. Riba et al. 1184 (UAMIZ); R. Riba et al. 1982 (UAMIZ). Tatahuicapan de Juárez: T. Krömer et al. 3587 (UAMIZ). Tlacotepec de Mejía, C. A. Purpus 1966 (MO). Totutla: F. Ventura A. 5300 (ENCB, XALU). Zongolica: T. Krömer et al. 03226 (MEXU).

Vittaria lineata (L.) Sm.

Córdoba: C. M. Rowell Jr. et al. 17M6 (MEXU). Naranjal: M. T. O. Benítez S. (CORU). San Andrés Tuxtla: J. H. Beaman y Álvarez 5768 (UAMIZ). Tlacotalpan: M. Nee y K. Taylor 29183 (NY). 


\section{APÉNDICE 2}

Helechos epífitos de Veracruz, para los que no se localizó el material de respaldo.

\section{ASPLENIACEAE}

Asplenium theciferum (Kunth) Mett.

(=Loxoscaphe thecifera $($ Kunth) T. Moore)

Ver. Bourgeau 3611 (i?), citado por Mickel \& Smith, 2004; Tejero-Diez et al., 2011.

Asplenium tuerckheimii Maxon

Ver. Córdoba: Finck 122 (UC), citado por Palacios-Rios, 1992. Totutla: C. A. Purpus 4347, 6432 (UC), citado por Palacios-Rios, 1992. Finck 41, 135 (UC); C. A. Purpus 6432 (UC), 4339 (UC, US), citado por Mickel \& Smith, 2004.

\section{DRYOPTERIDACEAE}

Elaphoglossum auricomum (Kunze) T. Moore

Ver. Hernández M.1385 (MEXU); Lira 227 (XAL); Müller 386 (NY), citados por Lira y Riba, 1984; Mickel \& Smith 2004; Tejero Diez et al., 2011.

Elaphoglossum ellipticifolium A. Rojas

Ver. Yecuatla: M. Nee et al. 26368 (F), citado por Rojas-Alvarado, 2003, Tejero-Diez et al. 2011. Elaphoglossum mesoamericanum A. Rojas

Ver. G. Castillo \& F. Vázquez 1469 (F), C. Conzatti 798 (GH), H. Fink 107 (MO), C. Purpus 1913 (MO), citados por Rojas-Alvarado, 2003, Tejero-Diez et al. 2011.

Elaphoglossum revolutum (Liebm.) T. Moore

Ver. P. E. Valdivia Q. 942 (XAL), citado por Mickel \& Smith, 2004; Tejero-Diez et al., 2011. Elaphoglossum setosum (Liebm.) T. Moore

Ver. Barranca de Huaitamalco, Liebman s.n. (US, P, K) (C!), citado por Mickel \& Smith, 2004; Tejero-Díez et al., 2011.

Elaphoglossum squamipes (Hook.) T. Moore

Ver. Cházaro y Castillo Ch. 6791 (IEB); Müller 37 (K), 39 (NY), citados por Mickel \& Smith, 2004; Tejero-Diez et al., 2011.

Elaphoglossum tectum (Humb. \& Bonpl. ex Willd.) T. Moore

Ver. Fortín de las Flores, Orizaba, Totutla y Xalapa, citado por Palacios-Rios, 1992; Müller 42 (NY) C. A. Purpus 2350, 6187 (US), citados por Mickel \& Smith 2004.

Elaphoglossum tejeroanum A. Rojas

Ver. J. Beaman \& C. Álvarez 5693 (MEXU), citado por Rojas-Alvarado, 2003, Tejero-Díez et al. 2011.

Elaphoglossum variabile A. Rojas

Ver. D. Barrington 428 (MEXU), citado por Rojas-Alvarado, 2003, Tejero-Díez et al. 2011.

Elaphoglossum xanthopodum Mickel

Ver. C. A. Purpus 16580 (US), Riba et al. 403 (NY), citados por Mickel \& Smith, 2004

HYMENOPHYLLACEAE

Hymenophyllum hirsutum (L.) Sw. 
Ver. M. Nee 24947 (XAL), citado por Mickel \& Smith, 2004; Tejero-Díez et al., 2011.

Trichomanes angustatum Carmich.

Ver. Correll y Correll 28768 (MICH, TEX); C. A. Purpus 2411 (UC), citados por Mickel \& Smith, 2004; Tejero-Diez et al., 2011.

Trichomanes tuerckheimii Christ

Ver. Gilly y Hernández X 44 (US); Sánchez 44 (US), citados por Mickel \& Smith, 2004;

Tejero-Diez et al., 2011.

POLYPODIACEAE

Campyloneurum costatum (Kunze) C. Presl

Ver. Vera-Santos 3010 (US), citado por Mickel \& Smith, 2004; Tejero-Diez et al., 2011.

Campyloneurum ensifolium (Willd.) J. Sm.

Ver. Barnett et al 85a (MO); Matuda 208 (MO); M. Nee y Hansen 18570 (F), citados por Mickel \& Smith 2004.

Campyloneurum tenuipes Maxon

Ver. Conzatti 590 (MEXU), citado por Mickel \& Smith, 2004; Tejero-Diez et al., 2011.

Melpomene moniliformis Smith \& Moran

Ver. Calcahualco: J. L. Martínez \& F. Vázquez 820 (XAL). Las Vigas de Ramírez: H. Narave F. y R. Ortega O. 599 (XAL). Orizaba?: F. Mueller 29 (NY). Xico: M. Palacios R. 2746 (XAL). Zacualpan: R. Ortega O. et al. 2669 (XAL), citados por Krömer et al., 2013a.

Melpomene peruviana (Desv.) A. R. Sm. \& R. C. Moran

Ver. Perote: J. H. Beaman 2173 (MEXU, UC, US), citado por Krömer et al., 2013a.

Micropolypodium taenifolium (Jenman) A. R. Sm.

(=Moranopteris taenifolia R. Y. Hirai \& J. Prado)

Ver: M. Nee 24971 (XAL); Vázquez et al 4108 (XAL), citados por Mickel \& Smith, 2004;

Tejero-Diez et al., 2011; Krömer et al. 2013.

Polypodium eperopeutes Mickel \& Beitel

Zongolica: T. Krömer 3074, citado por Viccon-Esquivel, 2009.

Ver. Copeland herb 133 (US); Croat 39547 (UC); F. Ventura A. 4904 (NY), citados por Mickel \& Smith, 2004; Tejero-Diez et al., 2011.

Terpsichore delicatula (M. Martens \& galeotti) A. R. Sm.

(=Galactodenia delicatula Sundue \& Labiak, Sundue et al. 2012: 340)

Ver. Orizaba?: F. Mueller 137 (NY); Vigas de Ramírez: L. Bohs et al. 1777 (MEXU, XAL), citados por Mickel \& Smith, 2004; Krömer et al., 2013a.

Terpsichore semihirsuta (Klotzsch) A. R. Sm.

Calcahualco: M. Nee 23139 (F, XAL), citado por Mickel \& Smith, 2004, Tejero-Diez et al., 2011; Krömer et al., 2013a.

Terpsichore spathulata A.R. Sm.

(=Alansmia spathulata Moguel \& M. Kessler)

Ver. M. Nee \& G. Diggs 24843 (MO, UC, XAL). Perote: H. Narave F. 823 (MEXU, XAL). Xico: H. Narave F. \& M. Nee 177 (XAL), citados por Mickel \& Smith, 2004; Krömer et al., 2013a. 


\section{APÉNDICE 3}

Helechos y licofitos epífitos ocasionales de Veracruz.

LYCOPODIACEAE

Lycopodiella cernua (L.) Pic. Serm.

Landero y Coss: P. E. Valdivia Q. 2209 (UAMIZ).

SELAGINELLACEAE

Selaginella flexuosa Spring.

Atzalan: T. Krömer 3477 (MEXU).

Selaginella martensii Sprig.

Banderilla: P. Hietz y U. Seifert 531 (XAL). Tlalnelhuayocan: A. Flores P. y J. G. García F. 899 (XAL). Xalapa: P. Hietz 36 (XAL).

DRYOPTERIDACEAE

Arachniodes denticulata (Sw.) Ching

Soteapan: R. Lira 6, 32 (UAMIZ, XAL). 